\title{
ENHANCEMENT OF THE TRAVELING FRONT SPEEDS IN REACTION-DIFFUSION EQUATIONS WITH ADVECTION
}

\author{
Alexander KISELEV, Leonid RYZHIK \\ Department of Mathematics, University of Chicago, Chicago, IL 60637, USA \\ Received 2 March 2000
}

\begin{abstract}
We establish rigorous lower bounds on the speed of traveling fronts and on the bulk burning rate in reaction-diffusion equation with passive advection. The non-linearity is assumed to be of either KPP or ignition type. We consider two main classes of flows. Percolating flows, which are characterized by the presence of long tubes of streamlines mixing hot and cold material, lead to strong speed-up of burning which is linear in the amplitude of the flow, $U$. On the other hand the cellular flows, which have closed streamlines, are shown to produce weaker increase in reaction. For such flows we get a lower bound which grows as $U^{1 / 5}$ for a large amplitude of the flow.

(C) 2001 L'Association Publications de l'Institut Henri Poincaré. Published by Elsevier B.V. All rights reserved

RÉSUMÉ. - Nous établissons rigoureusement une estimation inférieure de la vitesse de propagation de fronts et du taux global de combustion pour des équations de réactiondiffusion avec convection donnée. La non-linéarité est de type KPP ou de type "ignition". Nous considérons deux principales classes d'écoulements. Les écoulements de percolation, caractérisés par la présence de longs tubes de lignes de courant mélangeant des régions chaudes et froides, entraînent une accélération importante du taux de combustion, linéaire en fonction de l'intensité $U$ de l'écoulement. D'autre part, les écoulements circulaires, avec lignes de champs fermées, conduisent à une augmentation plus faible du taux de réaction. Pour de tels écoulements, nous obtenons une estimation inférieure qui croît comme $U^{1 / 5}$ pour des écoulements de grande amplitude. ๑ 2001 Éditions scientifiques et médicales Elsevier SAS
\end{abstract}

\section{Introduction}

Propagation of thin fronts in moving fluids arises in many situations in physics and engineering. Consider a mixture of reactants interacting in a region that may have a rather complicated spatial structure but is thin across. The reaction front moves towards the unburned reactants leaving behind the burned ones. When the reactants are mixed by an ambient fluid then the burning rate may be enhanced. The physical reason for this observed speed-up is believed to be that fluid advection tends to increase the area

E-mail addresses: kiselev@math.uchicago.edu (A. Kiselev), ryzhik@ math.uchicago.edu (L. Ryzhik). 
available for reaction. Many important engineering applications of combustion operate in the presence of turbulent advection, and therefore the influence of advection on burning has been studied extensively by physicists, engineers and mathematicians. In the physical literature one can find a number of models and approaches that yield different predictions - relations between the turbulent intensity and the burning rate $[8,19,20,34]$. These results are usually obtained using heuristic models and physical reasoning. For a recent review of some of the physics literature we refer to [27,29].

The lack of agreement between different physical models makes rigorous results, even for simplified mathematical models, particularly valuable and useful. A well-established mathematical model that describes a chemical reaction in a fluid is a system of two equations for concentration $C$ and temperature $T$ of the form

$$
\begin{aligned}
& T_{t}+u \cdot \nabla T=\kappa \Delta T+\frac{v_{0}^{2}}{\kappa} g(T) C, \\
& C_{t}+u \cdot \nabla C=\frac{\kappa}{\mathrm{Le}} \Delta C-\frac{v_{0}^{2}}{\kappa} g(T) C .
\end{aligned}
$$

For exposition purposes, all consideration in this paper will be carried out in two spacial dimensions, but our methods extend to an arbitrary dimension in a straightforward way. Eqs. (1.1) are coupled to the reactive Euler equations for the advection velocity $u(x, y, t)$. Two assumptions are usually made to simplify the problem: first, constant density approximation [8] that allows to decouple the Euler equations from the system (1.1). Then one may consider $u(x, y, t)$ as a prescribed quantity that does not depend on $T$ and $C$. Furthermore, it is often assumed that $\mathrm{Le}=1$, or, equivalently, thermal and material diffusivities are equal. These two assumptions allow to reduce the above system to a single scalar equation for the temperature $T$ :

$$
\frac{\partial T}{\partial t}+u(x, y, t) \cdot \nabla T=\kappa \Delta T+\frac{v_{0}^{2}}{\kappa} f(T)
$$

with $f(T)=g(T)(1-T)$, provided that $C(x, y, 0)=1-T(x, y, 0)$. We will consider the problem (1.2) in a strip $\Omega=\mathbb{R}_{x} \times[0, H]_{y}$ with boundary conditions in $x$ :

$$
T(x, y, t) \rightarrow 1 \quad \text { as } x \rightarrow-\infty, \quad T(x, y, t) \rightarrow 0 \quad \text { as } x \rightarrow+\infty
$$

and either Neumann

$$
\frac{\partial T}{\partial y}(x, 0, t)=\frac{\partial T}{\partial y}(x, H, t)=0,
$$

or periodic

$$
T(x, y, t)=T(x, y+H, t)
$$

boundary conditions in $y$. Furthermore, we assume that the initial data $T_{0}(x, y)$ for (1.2) satisfies the bounds

$$
\begin{aligned}
& T_{0}(x, y)=1-\mathrm{O}\left(\mathrm{e}^{\lambda x}\right) \quad \text { for } x<0, \quad T_{0}(x, y)=\mathrm{O}\left(\mathrm{e}^{-\lambda x}\right) \text { for } x>0, \\
& \left|\nabla T_{0}\right|=\mathrm{O}\left(\mathrm{e}^{-\lambda \mid x}\right) \quad \text { for some } \lambda>0 .
\end{aligned}
$$


We adopt fairly general assumptions on $f$, requiring only that $f(T)$ is not equal identically to zero, and

$$
f(0)=f(1)=0, \quad f(T) \geqslant 0 \quad \text { for } T \in(0,1), \quad f \in C^{1}[0,1] .
$$

Two types of reaction rates $f(T)$ are distinguished in this class. The KPP-type reactions satisfy

$$
f(0)=f(1)=0, \quad f(T)>0 \quad \text { for } T \in(0,1) .
$$

An additional requirement $f^{\prime}(0)=\max _{T \in[0,1]} f(T) / T$ is often made. We do not make such requirement in this paper, and call the class described by (1.9) general KPP. Our interpretation of KPP includes an important Arrhenius-type non-linearity,

$$
f(T)=C(1-T) \mathrm{e}^{-A / T},
$$

that is believed to be an appropriate model for many chemical reactions in the context of reaction-diffusion models. We also consider the ignition non-linearities with

$$
f(T)=0 \quad \text { for } T \in\left[0, \theta_{0}\right] \quad \text { and } \quad T=1, \quad f(T)>0 \quad \text { for } T \in\left(\theta_{0}, 1\right) .
$$

By our assumptions on the nonlinearity (1.8), we can find $\theta_{4}>\theta_{1}$, and $f_{0}, \zeta>0$ such that

$$
f(\theta)>f_{0} \quad \text { for } \theta \in\left(\theta_{1}-\zeta, \theta_{4}+\zeta\right) .
$$

The values of the constants $f_{0}, \zeta$ and $\theta_{1,4}$ are the only information on the nonlinearity $f(T)$ that shows up in our bounds on the burning rate.

We assume that advection $u(x, y) \in C^{1}(\Omega)$ is time independent, has mean zero in the $x$-direction:

$$
\int_{0}^{H} u_{1}(x, y) \mathrm{d} y=0
$$

and is incompressible:

$$
\nabla \cdot u=0
$$

The mathematical literature on the scalar reaction-diffusion equation (1.2) is enormous; far from giving an exhaustive overview, we mention several papers directly related to our work. First rigorous results about traveling waves for Eq. (1.2) go back to classical works of Kolmogorov, Petrovskii and Piskunov [23] and Fisher [13], which considered the case $u=0$ in one dimension for the KPP nonlinearity. Recently Eq. (1.2) with $u \neq 0$, and in particular the effect of advection, became a subject of intense research. Berestycki and Nirenberg [6,7], and Berestycki, Larrouturou and Lions [4] initiated the studies of the existence of traveling waves for Eq. (1.2) of the form

$$
T(x, y, t)=T(x-c t, y),
$$


for shear flows of the form $u=(u(y), 0)$. Their stability was studied in [5,25,30], while in further works $[3,31,32]$ stability and existence of traveling waves were established for the wider class of periodic flows. In this case, the traveling fronts have the form

$$
T(x, y, t)=U(x-c t, x, y)
$$

and are periodic in the last two variables. These and other results were recently reviewed in [33], and we refer the reader to this paper for a detailed exposition of the subject. Until very recently, there were no rigorous results on the physically interesting question of the speed of traveling waves. First such results have been established in [9] for percolating flows, and in [1] and [18] for the shear flows. Numerical studies of the propagation of fronts wore performed for a shear flow in [21] with $\mathrm{Le} \neq 1$, and for cellular flows in [22].

Another major direction of research has been homogenization approach. The homogenization regime $\kappa \rightarrow 0$, when the front width goes to zero, was extensively studied for KPP-type nonlinearity and for advection velocity that is periodic and varies either on the integral or diffusive scale by Freidlin [14-16]. Recently Majda and Souganidis derived an effective Hamilton-Jacobi equation in the limit $\kappa \rightarrow 0$ for the case of advection velocity varying on a small $\kappa$-dependent scale that is larger or comparable to that of the front width [24]. This effective equation is still difficult to analyze, and analytical predictions have been derived only for the shear flows. Numeric experiments exploring the results of [24] have been carried out in [10,11,26].

Very recently, Hamel [17] and Heinze, Stevens and Papanicolaou [18] proposed an elegant variational approach to the estimates of the speed of traveling waves in the presence of periodic advection. However, to the best of our knowledge nontrivial lower bounds using this method were obtained so far only for shear flows in the homogenization regime or for small advection, where they provide precise bounds for the small speed-up of the front [18].

The key question we wish to address in this paper is: what characteristics of the ambient fluid flow are responsible for burning rate enhancement? The question needs first to be made precise, because the reaction region may be complicated and, in general, may move with an ill-defined velocity, when traveling fronts do not exist. To measure the speed of burning in such situations, the bulk burning rate

$$
V(t)=\int_{\Omega} T_{t}(x, y, t) \frac{\mathrm{d} x \mathrm{~d} y}{H}
$$

and its time average

$$
\langle V\rangle_{t}=\frac{1}{t} \int_{0}^{t} V(s) \mathrm{d} s
$$

have been recently introduced in [9]. Note that for traveling fronts of the form (1.14) we have $V(t)=c$, but the notion of bulk burning rate makes sense in much more general situations when traveling fronts of the above form may not exist, and bulk burning rate serves as a natural generalization of the front speed. We have obtained in [9] lower bounds for $\langle V\rangle_{t}$ when $f(T)$ is a concave function of the KPP type. The 
bounds are linear in the magnitude of the advecting velocity $u(x, y)$ provided that there exist tubes of streamlines that connect $x=-\infty$ and $x=+\infty$, satisfying some mild additional technical assumptions. We say then that the flow is percolating. In particular these bounds hold for shear flows of the form $(u(y), 0)$.

In this paper we consider much more general reaction rates $f(T)$ that are either of the ignition or general KPP type, and establish similar lower bounds for $V(t)$ for percolating flows that are periodic in space. The bound is linear in the magnitude of $u$ and deteriorates as the scale of oscillations of $u$ becomes comparable to the laminar front width $l=\kappa / v_{0}$. It is easy to show [9] that for any $u \in C^{1}$, the burning rate $\langle V\rangle_{t}$ satisfies linear in $\|u\|_{\infty}$ upper bound (for initial data as in (1.6) and (1.7)). Therefore, shear (and, more generally, percolating) flows are as effective as possible in speeding up combustion in terms of the power of $\|u\|_{\infty}$ in the large intensity regime. In particular, we establish the following lower bound for the bulk burning rate in a shear flow.

THEOREM 1. - Let $T_{0}(x, y)$ be an arbitrary initial data satisfying (1.6) and (1.7), and let $T(x, y, t)$ satisfy (1.2) with either the Neumann (1.4) or periodic boundary conditions (1.5). Let also $u(x, y)=(u(y), 0)$ in (1.2). Then both for KPP and ignition non-linearities we have

$$
\lim _{t \rightarrow \infty}\langle V\rangle_{t} \geqslant C\left(v_{0}+\sum_{j}\left(1+\frac{l}{h_{j}}\right)^{-1} \int_{c_{j}-h_{j}}^{c_{j}+h_{j}}|u(y)| \frac{\mathrm{d} y}{H}\right),
$$

where the constant $C$ depends only on the reaction function $f$ and $l=k / v_{0}$. Here the intervals $I_{j}=\left[c_{j}-h_{j}, c_{j}+h_{j}\right] \in(0, H)$ are any intervals such that

$$
\frac{\|u\|_{\infty, j}}{2} \leqslant|u(y)| \leqslant\|u\|_{\infty, j}, \quad\|u\|_{\infty, j}=\sup _{I_{j}}|u(y)| .
$$

We do not require $\bigcup_{j} I_{j}=[0, H]$.

The choice of intervals $I_{j}$ is up to us, and should be made to maximize the lower bound. See Fig. 1 for an illustration.

As a corollary, the bound (1.16) holds for the speed $c$ of a traveling front of the from (1.14). Our bound behaves correctly in the homogenization regime when $u(y)$ has the form $u(y)=\frac{A}{\varepsilon} v(y / \varepsilon)$ and provides a bound that is linear in the magnitude $A$ of advection, in agreement with [9,18], where homogenization limit was studied. We also prove the analog of Theorem 1 for general percolating flows (see Theorem 5 in Section 3).

Another main result of this work concerns cellular flows with closed streamlines. Roughly speaking, in terms of their burning enhancement properties, such flows can be thought of as "the worst" class of flows, opposing "the best" percolating flows. One can expect the burning enhancement to be significantly weaker for cellular flows because of the numerous diffusive interfaces which prevent hot and cold regions from mixing fast. Cellular flows pose mathematically more challenging problem because of these diffusive interfaces; we will see that the estimates for percolating flows will form only a fraction 


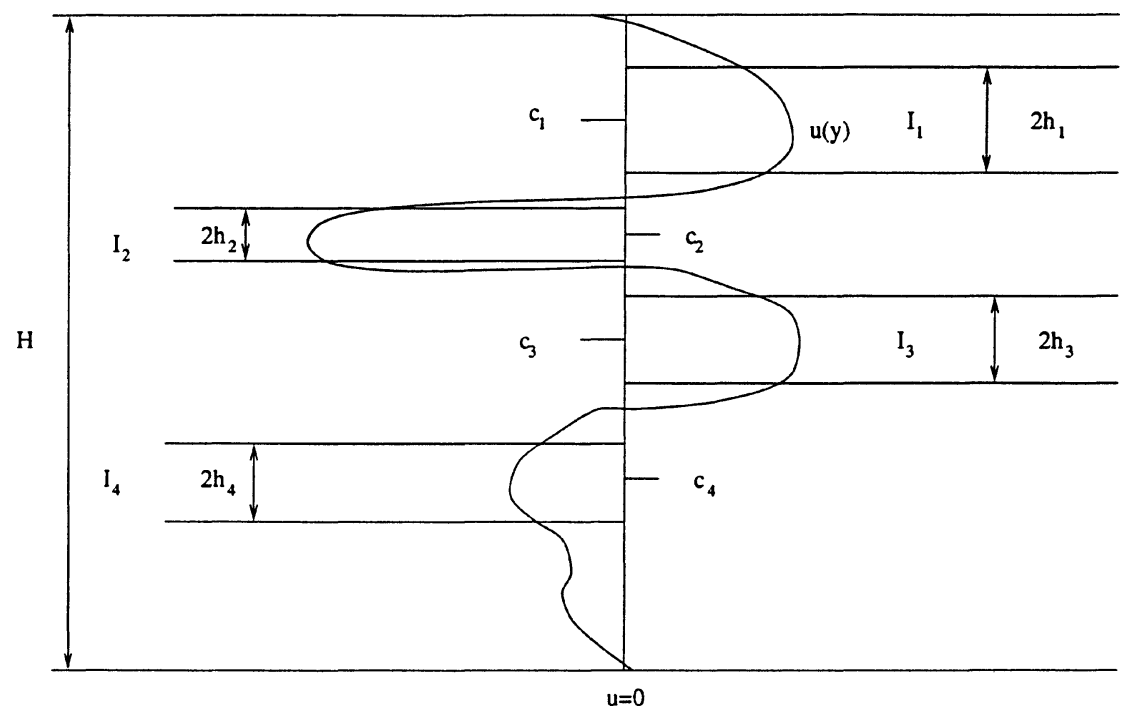

Fig. 1. The structure of the shear flow.

of the argument we will need in the cellular case. We consider a particular example of a cellular flow

$$
u(x, y)=U H\left(\frac{\partial \psi}{\partial y},-\frac{\partial \psi}{\partial x}\right), \quad \psi(x, y)=\sin \left(\frac{x}{H}\right) \sin \left(\frac{y}{H}\right) .
$$

See Fig. 2 for an illustration of streamlines in a single cell. Then under the assumptions of a large Peclet number and thin laminar front width:

$$
\mathrm{Pe}=\frac{U H}{\kappa} \geqslant 1, \quad \frac{l}{H} \leqslant 1, \quad l=\frac{\kappa}{v_{0}}
$$

we get the following result.

THEOREM 2. - Let $T_{0}(x, y)$ be an arbitrary initial data satisfying (1.6) and (1.7), and let $T(x, y, t)$ satisfy (1.2) with the either the Neumann (1.4) or periodic boundary conditions (1.5). Let also $u(x, y)$ in (1.2) be given by (1.18), and assume (1.19) is satisfied. Then both for KPP and ignition non-linearities we have

$$
\lim _{t \rightarrow \infty}\langle V\rangle_{t} \geqslant \begin{cases}\left(C_{1} \sqrt{\frac{\tau_{c}}{\tau_{u}}}+C_{2}\right) v_{0}, & \text { if } \tau_{c} \leqslant \tau_{u}, \\ \left(C_{1}\left(\frac{\tau_{c}}{\tau_{u}}\right)^{1 / 5}+C_{2}\right) v_{0}, & \text { if } \tau_{c} \geqslant \tau_{u} .\end{cases}
$$

Here $\tau_{c}=\kappa / v_{0}^{2}$ is the chemical reaction time and $\tau_{u}=H / U$ is the turnover time. The constants in the inequalities depend only on the reaction $f$, more particularly on the constants $f_{0}, \zeta$, and $\theta_{4}-\theta_{1}$ that appear in (1.11). 


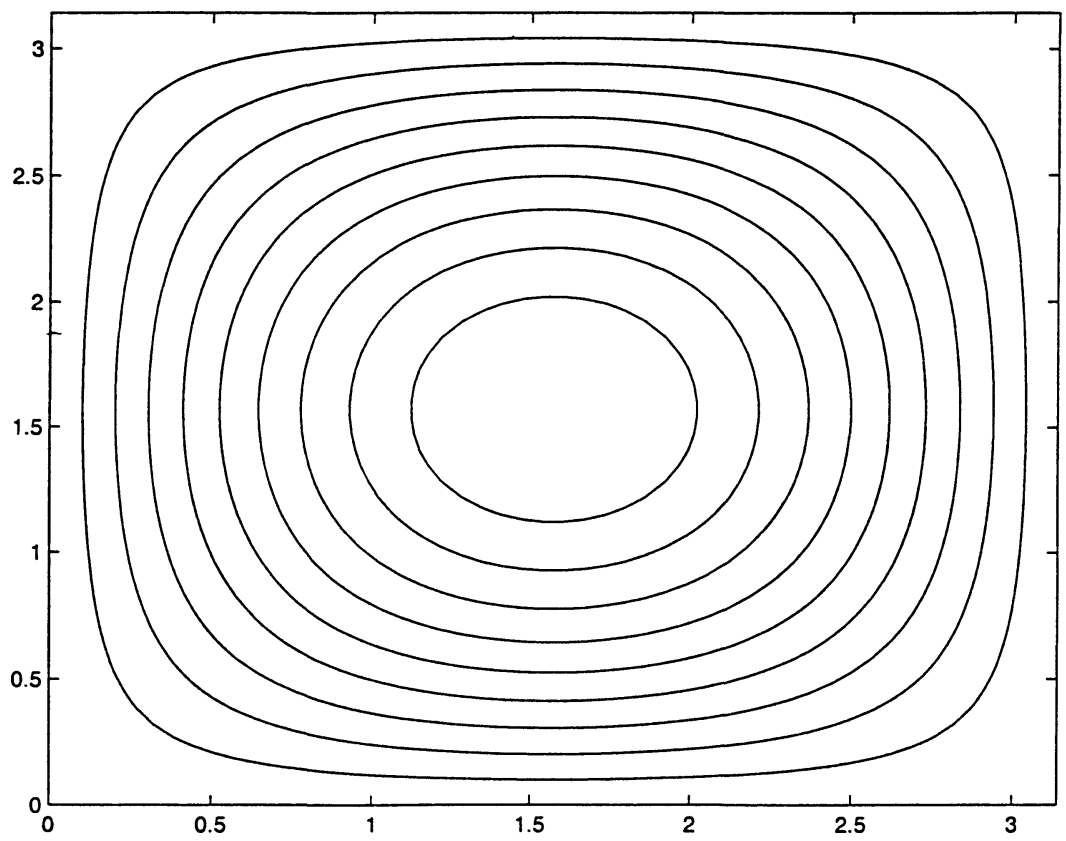

Fig. 2. Streamlines of a cellular flow $(H=\pi)$.

To the best of our knowledge this is the first rigorous bound on the traveling front speed in a cellular flow. Note that the change of behavior of our bound depending on the ratio $\tau_{c} / \tau_{u}$ is physically natural since for $\tau_{u} \ll \tau_{c}$ the front folds onto itself inside the period cell, which diminishes the affect of advection. The lower bound of Theorem 2 displays square root dependence on the flow intensity $U$ until $U$ reaches a critical value determined by a condition $\tau_{c}=\tau_{u}$. After that, the lower bound behaves like $U^{1 / 5}$. Recently, Audoly, Berestycki and Pomeau [1] gave an heuristic argument which proposes that the speed of the traveling front for cellular flows should scale as $U^{1 / 4}$ in the large $U$ limit, which may indicate that our lower bound is not far off from the sharp bound.

One of the fundamental mathematical difficulties we deal with in this paper may be roughly described as follows. We will be able to bound the burning rate from below by integrals over the domain of reaction term $f(T)$ and of the square of the gradient $|\nabla T|^{2}$. It turns out that in order to obtain a lower bound on $V$ in terms of $u$, it will be necessary to bound the integral of the higher derivative Laplacian term in terms of integrals of $f(T)$ and $|\nabla T|^{2}$. One can expect to do this using parabolic regularity, but the constants in such a priori estimates typically depend on $u$, and this dependence turns out to be too crude to get interesting results. We tackle this difficulty by taking advantage of the fact that what we need to estimate is the integral of Laplacian, not of the absolute value of Laplacian, and employ an appropriate averaging procedure to reduce derivatives. We hope that this idea will be useful in other related contexts in PDE estimates.

The paper is organized as follows. We prove Theorem 1 in Section 2, as well as some other results for shear flows. The analogous results for the percolating flows are proved in Section 3. We present our main results for the cellular flows, in particular implying 
Theorem 2, in Section 4. Sections 6, 7 and 8 contain some intermediate estimates in the proof of Theorem 2. We put these estimates together to finish the proof in Section 9.

\section{Shear flows and general nonlinearities}

We first consider (1.2) in a shear, or unidirectional, flow $(u(y), 0)$, which is a particular example of a percolating flow. The proofs are somewhat less technical in this case and allow us to introduce some of the ideas used in the general case. Eq. (1.2) in a shear flow becomes

$$
\begin{aligned}
& \frac{\partial T}{\partial t}+u(y) \frac{\partial T}{\partial x}=\kappa \Delta T+\frac{v_{0}^{2}}{\kappa} f(T), \\
& T(x, y, 0)=T_{0}(x, y) .
\end{aligned}
$$

The advection is assumed to be mean-zero:

$$
\int_{0}^{H} u(y) \mathrm{d} y=0 .
$$

We impose an additional assumption

$$
\frac{\partial T}{\partial t} \geqslant 0
$$

This condition is satisfied for all times provided that initially we have

$$
\kappa \Delta T_{0}-u(y) \frac{\partial T_{0}}{\partial x}+\frac{v_{0}^{2}}{\kappa} f\left(T_{0}\right) \geqslant 0,
$$

as follows from the maximum principle (see, e.g., [28]). Therefore (2.3) is not a constraint on the dynamics but rather on the initial data. We note that (2.3) is true for traveling fronts of the form $T(x-c t, y)$ both for general KPP and ignition nonlinearity $[4,7]$. We assume the usual boundary conditions (1.3) at the left and right ends of the strip $\Omega$ and either Neumann (1.4) or periodic (1.5) boundary conditions at $y=0, H$. We also require that the initial data $T_{0}(x, y)$ satisfies (1.6) and (1.7). These conditions are preserved by evolution (see e.g. [9]) if the advecting velocity $u(y) \in C^{1}[0, H]$, that is, we have for each $t>0$ :

$$
\begin{aligned}
& 1-T(x, y, t) \leqslant C(t) \mathrm{e}^{\lambda x} \quad \text { for } x<0, \quad T(x, y, t) \leqslant C(t) \mathrm{e}^{\lambda x} \quad \text { for } x>0, \\
& |\nabla T(x, y, t)| \leqslant C(t) \mathrm{e}^{-\lambda|x|}
\end{aligned}
$$

provided that (1.6) and (1.7) hold initially.

Let $I_{j}=\left(c_{j}-h_{j}, c_{j}+h_{j}\right) \subset[0, H]$ be a collection of intervals satisfying (1.17). In particular $u(y)$ does not change sign on the intervals $I_{j}$. We do not require that $\bigcup_{j} I_{j}=[0, H]$. Then the bulk burning rate $V(t)$ defined by (1.15) obeys a lower bound described by the following theorem, which is the first main result of this section. 
THEOREM 3. - Let $T(x, y, t)$ be a solution of (2.1) with the boundary conditions (1.3) and either (1.4) or (1.5). Let the initial data $T_{0}(x, y)$ satisfy (2.4), (1.6) and (1.7). Furthermore, assume that $u(y) \in C^{1}(0, H)$ has mean zero (2.2) and the nonlinearity $f(T)$ satisfies (1.8). Then there exists a constant $C>0$ that depends on $f(T)$ but not on $T_{0}(x, y)$ or $u(y)$, such that for any collection of intervals $I_{j}$ that satisfies $(1.17)$ we have

$$
V(t) \geqslant C\left(v_{0}+\sum_{j}\left(1+\frac{l}{h_{j}}\right)^{-1} \int_{c_{j}-h_{j}}^{c_{j}+h_{j}}|u(y)| \frac{\mathrm{d} y}{H}\right)
$$

with $l=\kappa / v_{0}$.

Remark 1. - The lower bound (2.6) does not deteriorate when oscillations in $u(y)$ become faster in space as long as its amplitude grows according to

$$
\frac{\|u\|_{\infty, j}}{v_{0}}=\mathrm{O}\left(\frac{l}{h_{j}}\right) \text {. }
$$

This agrees well with the homogenization limit $u_{\varepsilon}(y)=\frac{1}{\varepsilon} u(y / \varepsilon)$ considered in [9] and [18], that produces speed-up of the front of order $\mathrm{O}\left(v_{0}\right)$. This is also an improvement of the analogous lower bound for $V(t)$ for the convex KPP case obtained in [9], where $\left(l / h_{j}\right)^{2}$ appeared in the factor.

2. The regularity assumption on $u(y)$ is used only to guarantee preservation of the boundary conditions (1.7) that allows us to integrate by parts in the proof. None of our bounds depend on the size of derivatives of $u(y)$.

It has been shown in $[4,7]$ both in the case of ignition non-linearity $(1.10)$, and for the general KPP nonlinearity (1.9) that there exist traveling front solutions of (2.1) of the form $T(x, y, t)=U(x-c t, y)$. The speed $c=c_{*}$ is uniquely determined by the nonlinearity $f(T)$ and advection $u(y)$ in the ignition case, while traveling front solutions exist for $c \geqslant c_{u}$ for some minimal speed $c_{u}$ in the $\operatorname{KPP}$ case. The function $U(s, y)$ is monotonically decreasing in the variable $s=x-c t$ in both cases, so that (2.3) holds. Theorem 3 implies the following estimate on the speeds of the traveling fronts.

COROLLARY 1. - Let $T(x-c t, y)$ be a traveling front solution of $(2.1)$ with $f(T)$ being either of the ignition nonlinearity type (1.10), or of the KPP type (1.9). Then there exists a constant $C>0$ that depends on the function $f$ but not on $u(y)$ such that

$$
c \geqslant C\left(v_{0}+\sum_{j}\left(1+\frac{l}{h_{j}}\right)^{-1} \int_{c_{j}-h_{j}}^{c_{j}+h_{j}}|u(y)| \frac{\mathrm{d} y}{H}\right) .
$$

Corollary 1 follows immediately from Theorem 3 since we have $V(t)=c$ for $T(x, y, t)=U(x-c t, y)$ due to the boundary conditions $U(s, y) \rightarrow 1$ as $s \rightarrow-\infty$, $U(s, y) \rightarrow 0$ as $s \rightarrow+\infty$. Corollary 1 and the stability results for traveling fronts [30, 32] imply Theorem 1 for general initial data. We prove now Theorem 1 assuming the result of Corollary 1. 
Proof of Theorem 1. - Consider first ignition non-linearity. In this case we will show that

$$
\lim _{t \rightarrow \infty}\langle V\rangle_{t}=c_{*}
$$

where $c_{*}$ is the unique speed of the traveling front. Then (1.16) will follow from (2.7). It was shown in [32] that for the initial data satisfying (1.6) (actually just tending to 1 and 0 at the two ends) there exist functions $\xi_{1,2}(t)$ such that

$$
\left|\xi_{i}(t)\right|=\mathrm{o}(t), \quad \text { as } t \rightarrow \infty
$$

and functions $q_{i}(t, x)$ that satisfy the linearized problem

$$
\frac{\partial q_{i}}{\partial t}+u(y) \frac{\partial q_{i}}{\partial x}=\Delta q_{i}
$$

such that

$$
\begin{aligned}
& U\left(x-c_{*} t+\xi_{1}(t), y\right)-q_{1}(x, y, t) \\
& \quad \leqslant T(t, x, y) \leqslant U\left(x-c_{*} t-\xi_{2}(t), y\right)+q_{2}(x, y, t) .
\end{aligned}
$$

Here $U\left(x-c_{*} t, y\right)$ is the traveling wave solution of (2.1). The initial data $q_{i}(x, y, 0)$ may be chosen in $L^{1} \cap L^{\infty}(\Omega)$. Then we have for any $c>c_{*}$ :

$$
\begin{aligned}
\langle V\rangle_{\tau}= & \frac{1}{\tau} \int_{0}^{\tau} \mathrm{d} t \int_{\Omega} \frac{\mathrm{d} x \mathrm{~d} y}{H} T_{t} \mathrm{~d} x \mathrm{~d} y=\frac{1}{\tau} \int_{\Omega} \frac{\mathrm{d} x \mathrm{~d} y}{H}\left[T(x, y, \tau)-T_{0}(x, y)\right] \\
= & \frac{1}{\tau} \int_{-\infty}^{0} \mathrm{~d} x \int_{0}^{H} \frac{\mathrm{d} y}{H}\left[\left(1-T_{0}\right)-(1-T)\right]+\frac{1}{\tau} \int_{0}^{c \tau} \mathrm{d} x \int_{0}^{H} \frac{\mathrm{d} y}{H}\left[T(x, y, \tau)-T_{0}(x, y)\right] \\
& +\frac{1}{\tau} \int_{c \tau}^{\infty} \mathrm{d} x \int_{0}^{H} \frac{\mathrm{d} y}{H}\left[T(x, y, \tau)-T_{0}(x, y)\right] \\
\leqslant & \frac{C}{\tau}+c+\frac{1}{\tau} \int_{\left(c-c_{*}\right) \tau}^{\infty} \mathrm{d} x \int_{0}^{H} \frac{\mathrm{d} y}{H} U\left(x-\xi_{2}(t), y\right)+\frac{1}{\tau} \int_{c \tau}^{\infty} \mathrm{d} x \int_{0}^{H} \frac{\mathrm{d} y}{H} q_{2}\left(x+c_{*} t, y, t\right) \\
\leqslant & \frac{C^{\prime}}{\tau}+c
\end{aligned}
$$

and hence $\lim \sup _{\tau \rightarrow \infty}\langle V\rangle_{\tau} \leqslant c_{*}$. Similarly one may show that $\liminf _{\tau \rightarrow \infty}\langle V\rangle_{\tau} \geqslant c^{\prime}$ for any $c^{\prime}<c_{*}$, which shows that (1.16) holds.

In the KPP case, the estimates of the sort (2.8) are not yet available. However the bound (1.16) can be shown by reduction to the ignition non-linearity case. Indeed, given KPP type reaction $f$, consider ignition type reaction $f_{\theta}=\chi_{\theta} f \leqslant f$, say by cutting $f$ off in a small neighborhood near zero for $T \leqslant \theta$. The constant $C$ in (2.6) does not depend on $\theta$ for $\theta$ small enough as will be seen from the proof of Theorem 3. Let $T$ and $T_{\theta}$ satisfy equations with reactions $f$ and $f_{\theta}$ respectively with the same initial data $T_{0}(x, y)$. Then 
$Z=T-T_{\theta}$ satisfies

$$
Z_{t}+u \cdot \nabla Z-\kappa \Delta Z=\frac{r_{0}^{2}}{\kappa}\left(f(T)-f_{\theta}\left(T_{\theta}\right)\right) \geqslant \frac{v_{0}^{2}}{\kappa}\left(f(T)-f\left(T_{\theta}\right)\right) .
$$

It follows from the maximum principle that if $Z(x, 0) \geqslant 0$ then $Z(x, t) \geqslant 0$. Hence for the same initial data, the burning rate for the KPP reaction $f$ is not smaller than for the ignition non-linearity $f_{\theta}$ :

$$
\begin{aligned}
\langle V[T]\rangle_{t} & =\frac{1}{\tau} \int \frac{\mathrm{d} x \mathrm{~d} y}{H}\left[T(x, y, t)-T_{0}(x, y)\right] \\
& \geqslant \frac{1}{\tau} \int \frac{\mathrm{d} x \mathrm{~d} y}{H}\left[T_{\theta}(x, y, t)-T_{0}(x, y)\right]=\left\langle V\left[T_{\theta}\right]\right\rangle_{t} .
\end{aligned}
$$

This implies the validity of the lower bound (1.16) in the KPP case.

We now turn to the proof of Theorem 3. The proof follows the general ideas of [9] with significant modifications required since $f$ is not of concave KPP class. Our starting point is the following observation.

LEMMA 1. - Under assumptions of Theorem 3 we have

$$
V(t)=\frac{v_{0}^{2}}{\kappa} \int_{\Omega} f(T(x, y, t)) \frac{\mathrm{d} x \mathrm{~d} y}{H} \geqslant \kappa \int_{\Omega}|\nabla T(x, y, t)|^{2} \frac{\mathrm{d} x \mathrm{~d} y}{H} .
$$

Proof. - Equality in (2.9) is obtained simply by integrating (2.1) over $\Omega$ using the boundary conditions (1.3) and (1.4) or (1.5), and mean-zero condition (2.2) on advection. To get the inequality we multiply (2.1) by $T$ and integrate over $\Omega$ to get

$$
\int_{\Omega} T T_{t} \frac{\mathrm{d} x \mathrm{~d} y}{H}+\kappa \int_{\Omega}|\nabla T(x, y, t)|^{2} \frac{\mathrm{d} x \mathrm{~d} y}{H}=\frac{v_{0}^{2}}{\kappa} \int_{\Omega} T f(T) \frac{\mathrm{d} x \mathrm{~d} y}{H} \leqslant V(t) .
$$

This implies (2.9) since $T_{t} \geqslant 0$ and $0 \leqslant T \leqslant 1$.

As a warm-up, we now prove a simple and general proposition, which already provides a glimpse of some of the ideas which we will use to obtain more sophisticated results. Namely, we show that for any divergence-free velocity $u(x, y)$ satisfying mild regularity conditions (it does not have to be a shear flow), and solution $T$ satisfying (2.3), the burning rate is bounded below by $C v_{0}$.

Proposition 1. - Let $T(x, y, t)$ be a solution of (1.2) with the boundary conditions (1.3) and either (1.4) or (1.5). Assume that $u(x, y) \in C^{1}([0, H] \times \mathbb{R})$ satisfies $(1.12)$ and (1.13), and that non-linearity $f(T)$ satisfies (1.8). Let the initial data $T_{0}(x, y)$ satisfy (2.4), (1.6) and (1.7). Then there exists a constant $C$, depending only on the parameters $\zeta$ and $f_{0}$, such that

$$
V(t) \geqslant C\left(\zeta, f_{0}\right) v_{0}
$$

with the constants $\zeta$ and $f_{0}$ defined in (1.11). 
Proof. - The proof is similar to the proof of Lemma 2 in [9]. We can find $y$ such that

$$
\int_{\mathbb{R}}|\nabla T(x, y)|^{2} \mathrm{~d} x \leqslant \frac{3}{H} \int_{0}^{H} \int_{\mathbb{R}}\left|\nabla T\left(x, y^{\prime}\right)\right|^{2} \mathrm{~d} x \mathrm{~d} y^{\prime},
$$

and

$$
\int_{\mathbb{R}} f(T(x, y)) \mathrm{d} x \leqslant \frac{3}{H} \int_{0}^{H} \int_{\mathbb{R}} f\left(T\left(x, y^{\prime}\right)\right) \mathrm{d} x \mathrm{~d} y^{\prime} .
$$

Then we can find $x_{1} \leqslant x_{2}$ such that $T\left(x_{1}, y\right)=\theta_{4}+\zeta, T\left(x_{2}, y\right)=\theta_{1}-\zeta, T(x, y) \in$ $\left[\theta_{1}-\zeta, \theta_{4}+\zeta\right]$ if $x_{1} \leqslant x \leqslant x_{2}$ (see (1.11) for the definition of $\left.\theta_{1,4}\right)$. Then

$$
\int_{\mathbb{R}}|\nabla T(x, y)|^{2} \mathrm{~d} x \geqslant \frac{\theta_{4}-\theta_{1}+2 \zeta}{\left|x_{2}-x_{1}\right|}
$$

and

$$
\int_{\mathbb{R}} f(T(x, y)) \mathrm{d} x \geqslant f_{0}\left|x_{2}-x_{1}\right|
$$

Therefore we have

$$
\sqrt{\int_{\mathbb{R}} \int_{0}^{H}|\nabla T|^{2} \mathrm{~d} x \mathrm{~d} y \int_{\mathbb{R}} \int_{0}^{H} f(T) \mathrm{d} x \mathrm{~d} y} \geqslant C f_{0}^{1 / 2} \zeta H .
$$

Hence we obtain

$$
\int_{\mathbb{R}} \int_{0}^{H}\left[\kappa|\nabla T|^{2}+\frac{v_{0}^{2}}{\kappa} f(T)\right] \frac{\mathrm{d} x \mathrm{~d} y}{H} \geqslant C f_{0}^{1 / 2} \zeta v_{0} .
$$

Then Proposition 1 follows from Lemma 1.

We now return to the shear flows. To obtain more precise bounds involving advection velocity $u(y)$, we will bound from below in terms of $u(y)$ either the integral of $f(T)$ or the $L^{2}$-norm of $|\nabla T|$, and use Lemma 1 . The general plan in [9] was to integrate over all axis in $x$, obtaining an equation with an explicit term $u(y)$ in it. We were able to bound the rest of the terms from above by a combination of $\int f(T)$ and $\int|\nabla T|^{2}$ after averaging in $y$ and $t$ to control $T_{t}$ and $\Delta T$.

An additional twist we need here is to reduce our consideration to the region in space where the reaction actually takes place. In the case of ignition non-linearity, there is no reaction for sufficiently low temperatures. Similarly, for the Arrhenius type non-linearity, reaction is very weak near $T=0$. On the technical side, restriction of consideration to some finite time dependent domain $D$ with $T$ in appropriate range will mandate additional averaging in $x$ to control all terms by $\int f(T)$ and $\int|\nabla T|^{2}$. We will identify a 
region $D$ in $x$ such that on one hand the temperature has a certain drop over this region and on the other for every $x \in D$ there is some $y \in I_{j}$ such that reaction is bounded away from zero at the point $(x, y)$. This will provide us with two alternatives for each $x \in D$ : either reaction is uniformly bounded away from zero for that $x$ or temperature drops by a certain amount on the interval $x \times I_{j}$. In the first case $\int_{I_{j}} f(T) \mathrm{d} y$ will have to be large and in the second $\int_{I_{j}}\left|T_{y}\right|^{2} \mathrm{~d} y$ will be bounded from below. Then we will integrate (2.1) over $x \in D$ at a fixed time $t$. That will relate $u(y)$ to some terms involving $V(t)$ and $\Delta T$. We will additionally average both in $x$ and $y$, which will bring $\Delta T$ into a form that can be bound by a combination of integrals of $f(T)$ and $|\nabla T|^{2}$. That will be possible since these have to be large on $D$ as explained above. Finally we will use Lemma 1 to finish the proof.

In order to define the region $D$ where much of the reaction takes place let us fix $\theta_{4}>\theta_{3}>\theta_{2}>\theta_{1}$, where $\theta_{4}, \theta_{1}$ are as in (1.11). Let $I_{j}$ be an interval on which (1.17) holds with $u(y)>0$ (the case of $I_{j}$ where $u(y)<0$ is similar). We fix time $t>0$ and choose two points $x_{0}$ and $x_{1}$ :

$$
\begin{gathered}
x_{0}=\inf \left\{x: \text { for any } x^{\prime}>x \text { there exists } y \in I_{j} \text { such that } T\left(x^{\prime}, y, t\right) \leqslant \theta_{4}\right\}, \\
x_{1}=\sup \left\{x: x>x_{0} \text { and for any } x^{\prime} \in\left(x_{0}, x\right) \text { there exists } y \in I_{j}\right. \\
\text { such that } \left.T\left(x^{\prime}, y, t\right) \geqslant \theta_{1}\right\} .
\end{gathered}
$$

In other words, for any $x \in\left[x_{0}, x_{1}\right]=D$ there exists $y \in I_{j}$ such that $T(x, y, t) \in\left[\theta_{1}, \theta_{4}\right]$, and hence $f(T(x, y)) \geqslant f_{0}$. Note that $x_{0}$ is well-defined and finite since $T(x, y, t) \rightarrow$ 0,1 as $x \rightarrow \pm \infty$ uniformly in $x$ because of (2.5). The definition of $x_{0}$ implies that

$$
T\left(x_{0}, y\right) \geqslant \theta_{4} \text { for all } y
$$

and thus $x_{1}$ is well-defined. Moreover,

$$
T\left(x_{1}, y\right) \leqslant \theta_{1} \text { for all } y .
$$

In preparation for multiple averaging in $y$ that will be performed to control $\Delta T$ let us introduce the function $G(h, \xi)$ :

$$
G(h, \xi)= \begin{cases}1-\frac{|\xi|}{h}, & |\xi| \leqslant h, \\ 0, & |\xi|>h,\end{cases}
$$

that corresponds to the following averaging in $y$ :

$$
\frac{1}{h_{j}} \int_{0}^{h_{j}} \mathrm{~d} \delta \int_{c_{j}-\delta}^{c_{j}+\delta} p(y) \mathrm{d} y=\int_{c_{j}-h_{j}}^{c_{j}+h_{j}} G\left(h_{j}, y-c_{j}\right) p(y) \mathrm{d} y
$$

for a test function $p(y)$. The two integrations when applied to $\Delta T$ are required to get rid of derivatives of $T$. Observe that the function $G(h, \xi)$ has the following properties

$$
0 \leqslant G(h, \xi) \leqslant 1, \quad G(h, \xi) \geqslant \frac{1}{2} \quad \text { for } \xi \in\left[-\frac{h}{2}, \frac{h}{2}\right] .
$$


We note that (2.10) and (2.11) imply that at the two ends of the interval $\left[x_{0}, x_{1}\right]$ we have

$$
\begin{gathered}
\int_{c_{j}-h_{j}}^{c_{j}+h_{j}} \mathrm{~d} y G\left(h_{j}, y-c_{j}\right) u(y) T\left(x_{0}, y\right) \geqslant \theta_{4} F_{j}, \\
\int_{c_{j}-h_{j}}^{c_{j}+h_{j}} \mathrm{~d} y G\left(h_{j}, y-c_{j}\right) u(y) T\left(x_{1}, y\right) \leqslant \theta_{1} F_{j}
\end{gathered}
$$

with

$$
F_{j}=\int_{c_{j}-h_{j}}^{c_{j}+h_{j}} \mathrm{~d} y G\left(h_{j}, y-c_{j}\right) u(y) .
$$

In preparation for averaging in $x$, we choose $\eta_{0}$ and $\eta_{1}$ so that

$$
\begin{aligned}
& \eta_{0}=\inf _{\xi}\left\{\xi>0: \int_{c_{j}-h_{j}}^{c_{j}+h_{j}} \mathrm{~d} y G\left(h_{j}, y-c_{j}\right) u(y) T\left(x_{0}+\xi, y\right)=\theta_{3} F_{j}\right\}, \\
& \eta_{1}=\inf _{\xi}\left\{\xi>0: \int_{c_{j}-h_{j}}^{c_{j}+h_{j}} \mathrm{~d} y G\left(h_{j}, y-c_{j}\right) u(y) T\left(x_{1}-\xi, y\right)=\theta_{2} F_{j}\right\} .
\end{aligned}
$$

We remark that $x_{0}<x_{0}+\eta_{0}<x_{1}-\eta_{1}<x_{1}$. Now we are ready to average (2.1). Given $\alpha \in\left(0, \eta_{0}\right)$ and $\beta \in\left(0, \eta_{1}\right)$ we integrate $(2.1)$ in $x \in\left(x_{0}+\alpha, x_{1}-\beta\right)$ and in $y$ according to $(2.12)$ :

$$
\begin{gathered}
\int_{x_{0}+\alpha}^{x_{1}-\beta} \mathrm{d} x \int_{c_{j}-h_{j}}^{c_{j}+h_{j}} \mathrm{~d} y G\left(h_{j}, y-c_{j}\right) T_{t}-\kappa \int_{x_{0}+\alpha}^{x_{1}-\beta} \mathrm{d} x \int_{c_{j}-h_{j}}^{c_{j}+h_{j}} \mathrm{~d} y G\left(h_{j}, y-c_{j}\right) T_{y y} \\
\quad+\kappa \int_{c_{j}-h_{j}}^{c_{j}+h_{j}} \mathrm{~d} y G\left(h_{j}, y-c_{j}\right)\left[T_{x}\left(x_{0}+\alpha, y\right)-T_{x}\left(x_{1}-\beta, y\right)\right] \\
\geqslant \int_{c_{j}-h_{j}}^{c_{j}+h_{j}} \mathrm{~d} y G\left(h_{j}, y-c_{j}\right) u(y)\left[T\left(x_{0}+\alpha, y\right)-T\left(x_{1}-\beta, y\right)\right] .
\end{gathered}
$$

We dropped the integral of $f(T)$ on the right side which resulted in the inequality in (2.14). The reason that our averagings in $x$ and $y$ are different is that while the width $h_{j}$ is a prescribed quantity, we have no a priori control over $\eta_{0}$ and $\eta_{1}$. Therefore our bounds may not involve them, and we employ different estimates when averaging in $x$. First we estimate the integral of $T_{y y}$ on the left side of (2.14).

LEMMA 2. - There exists a universal constant $C>0$ such that the following estimate holds for every $x \in\left[x_{0}, x_{1}\right]$ 


$$
\begin{aligned}
& \left|\kappa \int_{c_{j}-h_{j}}^{c_{j}+h_{j}} \mathrm{~d} y G\left(h_{j}, y-c_{j}\right) T_{y y}(x, y)\right| \\
& \quad \leqslant C f_{0}^{-1 / 2} \zeta^{-1} \frac{\kappa}{v_{0} h_{j}}\left[\frac{v_{0}^{2}}{\kappa} \int_{c_{j}-h_{j}}^{c_{j}+h_{j}} \mathrm{~d} y f(T(x, y))+\kappa \int_{c_{j}-h_{j}}^{c_{j}+h_{j}} \mathrm{~d} y|\nabla T|^{2}(x, y)\right] .
\end{aligned}
$$

Proof. - We use (2.12) to rewrite the left side of (2.15) for a fixed $x \in\left[x_{0}, x_{1}\right]$ as

$$
\begin{aligned}
& \left|\int_{c_{j}-h_{j}}^{c_{j}+h_{j}} \mathrm{~d} y G\left(h_{j}, y-c_{j}\right) T_{y y}\right| \\
& \quad=\frac{1}{h_{j}}\left|T\left(c_{j}+h_{j}\right)-2 T\left(c_{j}\right)+T\left(c_{j}-h_{j}\right)\right| \leqslant \frac{2}{h_{j}} \delta_{j}[T](x)
\end{aligned}
$$

with $\delta_{j}[T](x)=\sup _{y \in I_{j}} T(x, y)-\inf _{y \in I_{j}} T(x, y)$. Note that because of our choice of $x_{0}$ and $x_{1}$, given any $x \in\left(x_{0}, x_{1}\right)$, we may find $y^{\prime}$ such that $T\left(x, y^{\prime}\right) \in\left[\theta_{1}, \theta_{4}\right]$. Then we may find $y_{1}, y_{2}$ such that for any $y \in\left[y_{1}, y_{2}\right]$ we have $T(x, y) \in\left(\theta_{1}-\zeta, \theta_{4}+\zeta\right)$, and, moreover,

$$
\left|T\left(x, y_{2}\right)-T\left(x, y_{1}\right)\right|=\min \left(\zeta, \delta_{j}[T](x)\right) .
$$

Then (1.11) implies that

$$
\int_{c_{j}-h_{j}}^{c_{j}+h_{j}} f(T(x, y)) \mathrm{d} y \geqslant f_{0}\left|y_{2}-y_{1}\right| .
$$

Applying the Cauchy-Schwartz inequality we also obtain

$$
\int_{c_{j}-h_{j}}^{c_{j}+h_{j}}|\nabla T(x, y)|^{2} \mathrm{~d} y \geqslant \frac{\left|T\left(x, y_{2}\right)-T\left(x, y_{1}\right)\right|^{2}}{\left|y_{2}-y_{1}\right|} .
$$

Multiplying these two inequalities we obtain

$$
\begin{aligned}
& \sqrt{\int_{c_{j}-h_{j}}^{c_{j}+h_{j}} f(T(x, y)) \mathrm{d} y \int_{c_{j}-h_{j}}^{c_{j}+h_{j}}|\nabla T(x, y)|^{2} \mathrm{~d} y} \\
& \geqslant \sqrt{f_{0}}\left|T\left(x, y_{2}\right)-T\left(x, y_{1}\right)\right| \geqslant \frac{f_{0}^{1 / 2} \zeta}{4}\left|T\left(c_{j}+h_{j}\right)-2 T\left(c_{j}\right)+T\left(c_{j}-h_{j}\right)\right|
\end{aligned}
$$

because of (2.16) and (2.17), and since $\zeta<1 / 2$. Then (2.15) follows. 
Furthermore, because of our choice of $\eta_{0}, \eta_{1}$, we have for any $\alpha \in\left[0, \eta_{0}\right]$ and $\beta \in\left(0, \eta_{1}\right)$

$$
\int_{c_{j}-h_{j}}^{c_{j}+h_{j}} \mathrm{~d} y G\left(h_{j}, y-c_{j}\right) u(y)\left[T\left(x_{0}+\alpha, y\right)-T\left(x_{1}-\beta, y\right)\right] \geqslant\left(\theta_{3}-\theta_{2}\right) F_{j} .
$$

We use (2.15), (2.18) and positivity of $T_{t}$ to rewrite (2.14) as

$$
\begin{aligned}
& C \int_{x_{0}+\alpha}^{x_{1}-\beta} \mathrm{d} x \int_{c_{j}-h_{j}}^{c_{j}+h_{j}} \mathrm{~d} y\left[T_{t}+\frac{\kappa}{v_{0} h_{j}}\left[\kappa|\nabla T|^{2}+\frac{v_{0}^{2}}{\kappa} f(T(x, y))\right]\right] \\
& \quad+\kappa \int_{c_{j}-h_{j}}^{c_{j}+h_{j}} \mathrm{~d} y G\left(h_{j}, y-c_{j}\right)\left[T_{x}\left(x_{0}+\alpha, y\right)-T_{x}\left(x_{1}-\beta, y\right)\right] \geqslant\left(\theta_{3}-\theta_{2}\right) F_{j} .
\end{aligned}
$$

In order to deal with the integral term on the second line that involves $T_{x}$ we average (2.19) in $\alpha \in\left(0, \eta_{0}\right)$ and $\beta \in\left(0, \eta_{1}\right)$ :

$$
\frac{1}{\eta_{0} \eta_{1}} \int_{0}^{\eta_{0}} \mathrm{~d} \alpha \int_{0}^{\eta_{1}} \mathrm{~d} \beta
$$

to get

$$
\begin{aligned}
& C \int_{x_{0}}^{x_{1}} \mathrm{~d} x \int_{c_{j}-h_{j}}^{c_{j}+h_{j}} \mathrm{~d} y\left[T_{t}+\frac{\kappa}{v_{0} h_{j}}\left[\kappa|\nabla T|^{2}+\frac{v_{0}^{2}}{\kappa} f(T(x, y))\right]\right] \\
& \quad+\frac{\kappa}{\eta_{0}} \int_{c_{j}-h_{j}}^{c_{j}+h_{j}} \mathrm{~d} y G\left(h_{j}, y-c_{j}\right)\left[T\left(x_{0}+\eta_{0}, y\right)-T\left(x_{0}, y\right)\right] \\
& \quad+\frac{\kappa}{\eta_{1}} \int_{c_{j}-h_{j}}^{c_{j}+h_{j}} \mathrm{~d} y G\left(h_{j}, y-c_{j}\right)\left[T\left(x_{1}-\eta_{1}, y\right)-T\left(x_{1}, y\right)\right] \geqslant\left(\theta_{3}-\theta_{2}\right) F_{j} .
\end{aligned}
$$

We bound now the term involving $\eta_{0}$ in (2.20) as follows.

LEMMA 3. - There exists a universal constant $C>0$ such that

$$
\begin{aligned}
& \left|\frac{\kappa}{\eta_{0}} \int_{c_{j}-h_{j}}^{c_{j}+h_{j}} \mathrm{~d} y G\left(h_{j}, y-c_{j}\right)\left[T\left(x_{0}+\eta_{0}, y\right)-T\left(x_{0}, y\right)\right]\right| \\
& \quad \leqslant C \kappa\left(\theta_{4}-\theta_{3}\right)^{-2} \int_{c_{j}-h_{j}}^{c_{j}+h_{j}} \mathrm{~d} y \int_{x_{0}}^{x_{0}+\eta_{0}} \mathrm{~d} x|\nabla T|^{2} .
\end{aligned}
$$


Proof. - The proof of this estimate is based on two observations. First, we have

$$
\left|\frac{1}{\eta_{0}} \int_{c_{j}-h_{j}}^{c_{j}+h_{j}} \mathrm{~d} y G\left(h_{j}, y-c_{j}\right)\left[T\left(x_{0}+\eta_{0}, y\right)-T\left(x_{0}, y\right)\right]\right| \leqslant \frac{2 h_{j}}{\eta_{0}}
$$

because of (2.13). Second, we have

$$
\int_{c_{j}-h_{j}}^{c_{j}+h_{j}} \mathrm{~d} y \int_{x_{0}}^{x_{0}+\eta_{0}} \mathrm{~d} x|\nabla T|^{2} \geqslant C\left(\theta_{4}-\theta_{3}\right)^{2} \frac{h_{j}}{\eta_{0}} .
$$

This bound is established as follows. Recall that because of our choice of $\eta_{0}$ we have

$$
\int_{c_{j}-h_{j}}^{c_{j}+h_{j}} \mathrm{~d} y u(y) G\left(h_{j}, y-c_{j}\right)\left[T\left(x_{0}, y\right)-T\left(x_{0}+\eta_{0}, y\right)\right] \geqslant\left(\theta_{4}-\theta_{3}\right) F_{j} .
$$

Furthermore, recall that $\|u\|_{\infty, j} / 2 \leqslant|u(y)| \leqslant\|u\|_{\infty, j}$ on the interval $\left(c_{j}-h_{j}, c_{j}+h_{j}\right)$ and thus (2.23) implies that

$$
\int_{c_{j}-h_{j}}^{c_{j}+h_{j}} \mathrm{~d} y G\left(h_{j}, y-c_{j}\right)\left|T\left(x_{0}, y\right)-T\left(x_{0}+\eta_{0}, y\right)\right| \geqslant \frac{\left(\theta_{4}-\theta_{3}\right) F_{j}}{\|u\|_{\infty, j}} \geqslant C\left(\theta_{4}-\theta_{3}\right) h_{j} .
$$

Then we obtain using the Cauchy-Schwartz inequality:

$$
\begin{aligned}
C\left(\theta_{4}-\theta_{3}\right) h_{j} \leqslant & \left(\int_{c_{j}-h_{j}}^{c_{j}+h_{j}} \mathrm{~d} y G^{2}\left(h_{j}, y-c_{j}\right)\right)^{1 / 2} \\
& \times\left(\int_{c_{j}-h_{j}}^{c_{j}+h_{j}} \mathrm{~d} y\left|T\left(x_{0}, y\right)-T\left(x_{0}+\eta_{0}, y\right)\right|^{2}\right)^{1 / 2} \\
\leqslant & C h_{j}^{1 / 2}\left(\int_{c_{j}-h_{j}}^{c_{j}+h_{j}} \mathrm{~d} y\left|\int_{x_{0}}^{x_{0}+\eta_{0}} \mathrm{~d} x T_{x}(x, y)\right|^{2}\right)^{1 / 2} \\
\leqslant & C h_{j}^{1 / 2}\left(\left.\eta_{c_{j}+h_{j}}^{x_{0}+\eta_{0}} \mathrm{~d} y \int_{c_{j}-h_{j}}^{x_{0}} \mathrm{~d} x T_{x}^{2}(x, y)\right|^{2}\right)^{1 / 2}
\end{aligned}
$$

and (2.22) follows.

A bound similar to (2.21) holds for the integral involving $\eta_{1}$ in (2.20). We use these two estimates in (2.20) to get 


$$
\begin{aligned}
& \int_{x_{0}}^{x_{1}} \mathrm{~d} x \int_{c_{j}-h_{j}}^{c_{j}+h_{j}} \mathrm{~d} y\left[T_{t}+\frac{\kappa}{v_{0} h_{j}}\left[\kappa|\nabla T|^{2}+\frac{v_{0}^{2}}{\kappa} f(T(x, y))\right]\right]+\kappa \int_{c_{j}-h_{j}}^{c_{j}+h_{j}} \mathrm{~d} y \int_{x_{0}}^{x_{1}} \mathrm{~d} x|\nabla T|^{2} \\
& \geqslant C F_{j} \geqslant C^{\prime} \int_{c_{j}-h_{j}}^{c_{j}+h_{j}} \mathrm{~d} y|u(y)| .
\end{aligned}
$$

A similar estimate holds also for the intervals $I_{j}$, on which $u(y)<0$. The only difference would be that at the first step of obtaining the analog of (2.14) one has to drop the integral involving $T_{t}$ and not that of $f(T)$. The rest of the estimates still hold. We use Lemma 1 in (2.24) to get after summation over all intervals $I_{j}$ :

$$
V(t) \geqslant C \sum_{j}\left(1+\frac{l}{h_{j}}\right)^{-1} \int_{c_{j}-h_{j}}^{c_{j}+h_{j}}|u(y)| \frac{\mathrm{d} y}{H},
$$

with $l=\kappa / v_{0}$. Finally, we can always add $v_{0}$ to the right hand side by Proposition 1 . This finishes the proof of Theorem 3.

\section{Percolating flows}

We now consider equation

$$
\begin{aligned}
& T_{t}+u(x, y) \cdot \nabla T=\kappa \Delta T+\frac{v_{0}^{2}}{\kappa} f(T), \\
& T(x, y, 0)=T_{0}(x, y),
\end{aligned}
$$

in a more general class of flows, which we call "percolating". By this we mean that there exist tubes of streamlines of the advecting velocity $u(x, y)$, which connect $x=-\infty$ and $x=+\infty$ in either direction, as depicted on Fig. 3. We assume that the flow has zero mean (1.12) and hence such tubes of streamlines will go in both directions. More precisely, let us assume that there exist regions $D_{j}^{+}$and $D_{J}^{-}, j=1, \ldots, N$, such that each of them is bounded by the streamlines of $u(x, y)$, and the projection of each streamline of $u(x, y)$, contained in either $D_{j}^{+}$or $D_{j}^{-}$, onto the $x$-axis covers the whole real line (these projections need not be one-to-one, however). We denote by $D_{ \pm}$the union of all $D_{j}^{ \pm}$respectively.

We will further assume that the velocity $u(x, y)$ is periodic in space. Then it is known $[3,31,32]$ that for ignition nonlinearity (1.10) there exist periodic traveling fronts. They have the form $T(x-c t, x, y)$ and are periodic in the last two variables and monotonically decreasing in the first one. These solutions satisfy our main condition

$$
\frac{\partial T}{\partial t}(x, y, t) \geqslant 0
$$



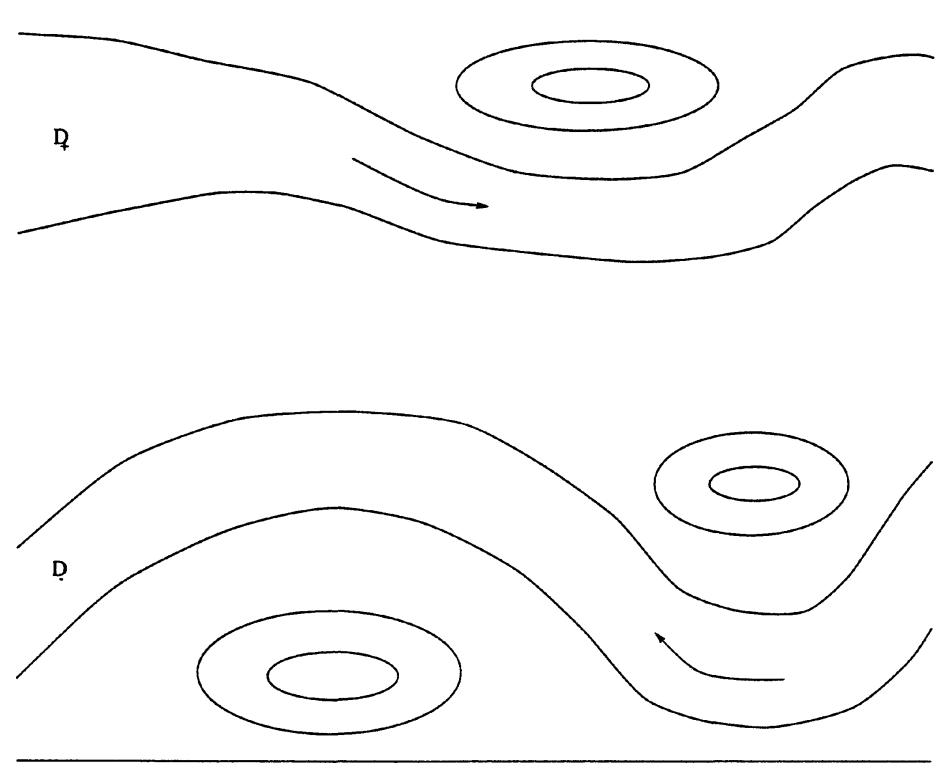

Fig. 3. Streamlines of $u(x, y)$.

Our results may be generalized in a straightforward manner to non-periodic percolating flows as long as initial data satisfies (3.2):

$$
\kappa \Delta T_{0}+\frac{v_{0}^{2}}{\kappa} f\left(T_{0}\right)-u \cdot \nabla T_{0} \geqslant 0 .
$$

However, we restrict our attention to periodic $u(x, y)$ to simplify the presentation.

We assume that the streamlines in $D_{j}^{ \pm}$are sufficiently regular, so that inside each $D_{j}^{ \pm}$there exists a one-to-one $C^{2}$ change of coordinates $(x, y) \rightarrow(\rho, \xi)$, such that $\rho$ is constant on the streamlines, while $\xi$ is an orthogonal coordinate for $\rho$ (with a slight abuse of notation we shall use the same notation $(\rho, \xi)$ in all $D_{j}^{ \pm}$, although these coordinates may not be defined globally). Moreover, $u \cdot \nabla \xi>0$ in $D_{j}^{+}$, while $u \cdot \nabla \xi<0$ in each $D_{j}^{-}$. The variable $\rho$ varies in the interval $\left[c_{j}^{ \pm}-h_{j}^{ \pm}, c_{j}^{ \pm}+h_{j}^{ \pm}\right]$, while $\xi$ varies in $(-\infty, \infty)$ in the set $D_{j}^{ \pm}$. See Fig. 4 for a sketch of coordinates $(\rho, \xi)$. The square of the length element inside each set $D_{j}^{ \pm}$is given by

$$
\mathrm{d} x^{2}+\mathrm{d} y^{2}=E_{1}^{2}(\rho, \xi) \mathrm{d} \rho^{2}+E_{2}^{2}(\rho, \xi) \mathrm{d} \xi^{2} .
$$

We assume that the functions $E_{1,2}$ are bounded from below:

$$
C^{-1} \leqslant E_{1,2}(\rho, \xi)
$$

uniformly on all $D_{j}^{ \pm}$. Moreover, the function

$$
\omega(\rho, \xi)=\frac{E_{2}(\rho, \xi)}{E_{1}(\rho, \xi)}
$$




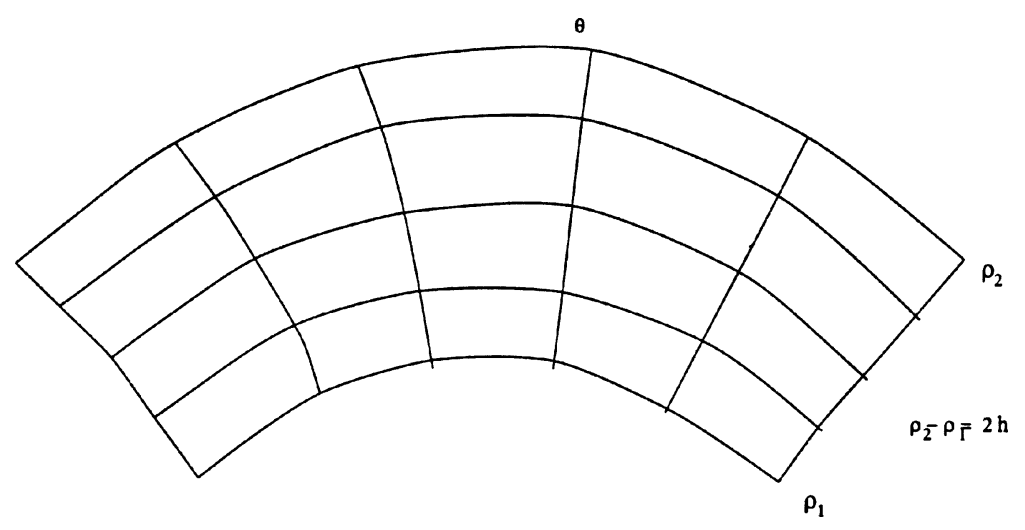

Fig. 4. Curvilinear coordinates $(\rho, \xi)$.

satisfies the following bounds:

$$
C^{-1} \leqslant|\omega(\rho, \xi)| \leqslant C, \quad\left|\frac{\partial \omega}{\partial \xi}(\rho, \xi)\right|, \quad\left|\frac{\partial \omega}{\partial \rho}(\rho, \xi)\right| \leqslant \frac{C}{h_{j}^{ \pm}} \quad \text { on } D_{j}^{ \pm} \text {, respectively, }
$$

with $2 h_{j}^{ \pm}$being the absolute value of the difference of the values of $\rho$ on the two components of the boundary $\partial D_{j}^{ \pm}$(recall that $D_{j}^{ \pm}$are bounded by two streamlines of $u(x, y))$. Finally we assume that the flux density $E_{1} u$ does not oscillate too much on the set $D_{j}^{ \pm}$:

$$
\begin{aligned}
& \frac{\left\|E_{1} u\right\|_{\infty, j}}{2} \leqslant E_{1}(\rho, \theta)|u(\rho, \theta)| \leqslant\left\|E_{1} u\right\|_{\infty, j} \quad \text { for }(x, y) \in D_{j}^{ \pm}, \\
& \left\|E_{1} u\right\|_{\infty, j}=\sup _{(\rho, \xi) \in D_{j}^{ \pm}}\left|E_{1}(\rho, \xi) u(\rho, \xi)\right| .
\end{aligned}
$$

Note that $E_{1}(\rho, \xi) \mid u(\rho, \xi)$ is independent of $\xi$. In particular if

$$
u(x, y)=U H \nabla^{\perp} \Psi=U H\left(\frac{\partial \Psi}{\partial y},-\frac{\Psi}{\partial x}\right)
$$

with $|\nabla \Psi| \leqslant C / H$ we may choose $\rho=H \Psi(x, y)$ so that $E_{1}=\frac{1}{H|\nabla \Psi|}$. Then we have $E_{1}|u|=U$, so that (3.7) holds automatically and (3.4) also holds for $E_{1}$. Other conditions on the streamlines may be also easily restated in terms of the stream function $\Psi(x, y)$.

We do not make any assumptions on the behavior of the streamlines of $u(x, y)$ outside the regions $D_{+}$and $D_{-}$. In particular, there may be pockets of still fluid, streamlines may be closed, etc. (see Fig. 3).

Then we have the following theorem.

THEOREM 4. - Let $T(x, y, t)$ be a solution of (3.1) with the boundary conditions (1.3) and (1.4) or (1.5), with the initial data $T_{0}(x, y)$ satisfying (3.3), and nonlinearity $f(T)$ satisfying (1.8). Let each of the sets $D_{j}^{ \pm}$be of the form $D_{j}^{ \pm}=\left\{\rho \in\left[c_{j}-h_{j}\right.\right.$, 
$\left.\left.c_{j}+h_{j}\right]\right\}$. Then under the assumptions (3.4) and (3.6) on the streamlines of the flow $u(x, y) \in C^{1}(\Omega)$, we have

$$
V(t) \geqslant C\left(v_{0}+\sum_{D_{j}^{ \pm}}\left(1+\frac{l}{h_{j}}\right)^{-1} \int_{c_{j}-h_{j}}^{c_{j}+h_{j}}|u(\rho, \xi)| E_{1}(\rho, \xi) \frac{\mathrm{d} \rho}{H}\right)
$$

for all $t>0$. Here $l=\kappa / v_{0}$ and the constant $C$ in (3.8) depends only on the function $f(T)$ and the constants appearing in (3.4) and (3.6).

Note that the integrals on the right side of (3.8) give fluxes of $u(x, y)$ through the tubes of the streamlines. As in the shear case the pre-factor $\left(1+l / h_{j}\right)^{-1}$ agrees with the homogenization limit $[9,18]$.

Recall that traveling fronts for periodic flows have the form

$$
T(x, y, t)=U(x-c t, x, y)
$$

with the function $U(s, x, y)$ being periodic in the last two variables. It was shown in $[3,31,32]$ that in the ignition nonlinearity case (1.10) such traveling fronts exist and their speed $c_{*}$ is unique. Their existence for the KPP nonlinearities (1.9) was shown recently in [3] with $c \geqslant c_{u}, c_{u}$ being the minimal traveling front speed. The following analog of Corollary 1 holds for percolating flows, which we formulate separately for the convenience of the reader.

COROLLARY 2. - Let $f(T)$ be either of the ignition nonlinearity type (1.10), or of the KPP type (1.9). Let also $U(x-c t, x, y)$ be a traveling wave-type solution of (3.1), periodic in the second two variables. Then there exists a constant $C>0$ that depends only on the function $f$ and on the constants appearing in (3.4) and (3.6) such that

$$
c \geqslant C\left(v_{0}+\sum_{j}\left(1+\frac{l}{h_{j}}\right)^{-1} \int_{c_{j}-h_{j}}^{c_{j}+h_{j}}|u(\rho, \xi)| E_{1}(\rho, \xi) \frac{\mathrm{d} \rho}{H}\right) .
$$

Proof. - Corollary 2 follows from Theorem 4 as follows. Let $U(x-c t, x, y)$ be a periodic traveling front solution of (3.1) such that

$$
U(s, x+L, y)=U(s, x, y)
$$

and let $\tau=L / c$. Then we have

$$
\begin{aligned}
\frac{1}{\tau} \int_{0}^{\tau} V(t) \mathrm{d} t & =\frac{1}{\tau} \int_{0}^{\tau} \mathrm{d} t \int_{\Omega}\left(-c U_{s}(x-c t, x, y)\right) \frac{\mathrm{d} x \mathrm{~d} y}{H} \\
& =\frac{1}{\tau} \int_{0}^{L} \mathrm{~d} t^{\prime} \int_{\Omega}\left[-U_{s}\left(x-t^{\prime}, x, y\right)\right] \frac{\mathrm{d} x \mathrm{~d} y}{H}
\end{aligned}
$$




$$
\begin{aligned}
& =-\frac{c}{L} \int_{0}^{L} \mathrm{~d} t^{\prime} \int_{\Omega}\left[\frac{\mathrm{d}}{\mathrm{d} x} U\left(x-t^{\prime}, x, y\right)-U_{x}\left(x-t^{\prime}, x, y\right)\right] \frac{\mathrm{d} x \mathrm{~d} y}{H} \\
& =c+\frac{c}{L} \int_{0}^{L} \mathrm{~d} t^{\prime} \int_{\Omega} U_{x}\left(x-t^{\prime}, x, y\right) \frac{\mathrm{d} x \mathrm{~d} y}{H} \\
& =c+\frac{c}{L} \int_{\Omega} \frac{\mathrm{d} x \mathrm{~d} y}{H}[U(x, x+L, y)-U(x, x, y)]=c .
\end{aligned}
$$

Remark. - We note that Corollary 2 implies a lower bound on the effective diffusivity [2] in the homogenization regime. Recall that solutions of the advection-diffusion equation (3.1) with $f(T)=0$, and with advection of the form $u(x, y)=\frac{U}{\varepsilon} v\left(\frac{x}{\varepsilon}, \frac{y}{\varepsilon}\right)$ with $v(x, y)$ periodic, converge as $\varepsilon \rightarrow 0$ to the solution $\bar{T}$ of the homogenized problem

$$
\frac{\partial \bar{T}}{\partial t}=\kappa_{i j}^{*} \frac{\partial^{2} \bar{T}}{\partial x_{i} \partial x_{j}}, \quad \bar{T}(x, y, 0)=T_{0}(x, y), \quad\left(x_{1}, x_{2}\right)=(x, y) .
$$

The effective diffusivity $k^{*}$ is a complicated functional of advection $U v(\mathbf{y})$. Explicit bounds on $\kappa^{*}$ in terms of the magnitude $U$ of advection are easy to obtain in the shear case, when $\kappa^{*}$ may be found explicitly, and effective diffusivity in the direction of the flow $\kappa_{x x}^{*} \sim U^{2}$. Using results of [18] one may deduce from Corollary 2 that this bound applies also to periodic percolating flows, despite the fact that no explicit expression for $\kappa^{*}$ is known in this case.

Corollary 2 and the stability results of [32] imply the analog of Theorem 1.

THeOREM 5. - Let the initial data $T_{0}(x, y)$ for Eq. (3.1) satisfy the decay to 0,1 conditions (1.6) and (1.7). Then both for KPP and ignition non-linearities we have

$$
\lim _{t \rightarrow \infty}\langle V\rangle_{t} \geqslant C\left(v_{0}+\sum_{j}\left(1+\frac{l}{h_{j}}\right)^{-1} \int_{c_{j}-h_{j}}^{c_{j}+h_{j}}|u(\rho, \xi)| E_{1}(\rho, \xi) \frac{\mathrm{d} \rho}{H}\right),
$$

where constant $C$ depends only on the non-linearity $f$ and on the constants appearing in (3.4) and (3.6).

Now we turn to the proof of Theorem 4. The proof is a modification of the proof of Theorem 3. We will again utilize Lemma 1 as well as averaging along the streamlines of $u(x, y)$ in order to bound the arising averages of $\Delta T$ in terms of integrals of $f(T)$ and $|\nabla T|^{2}$. The additional technical difficulties are due to the fact that two natural geometries of the problem - streamlines for the advective term and Euclidean coordinates for Laplacian - are in harmony in the case of shear flows, but at odds in the case of more general percolating flows. Moreover, while in the case of shear flows we gave the proof that we felt was simplest, here we will use the approach which is slightly more involved; however, it is better adapted for the application to cellular flows in the following sections.

Let us consider a region $D_{j}^{+}=\left\{(\rho, \xi): \rho \in\left[c_{j}-h_{j}, c_{j}+h_{j}\right]\right\}$ with $u \cdot \nabla \xi>0$. Introduce notation $k_{j}(\rho)=G\left(h_{j}, \rho-c_{j}\right) E_{1}(\rho, \xi)|u(\rho, \xi)|\left(k_{j}\right.$ does not depend on $\xi$ 
by incompressibility of $u(x, y))$ and, similarly to the shear case,

$$
F_{j}=\int_{c_{j}-h_{j}}^{c_{j}+h_{j}} k_{j}(\rho) \mathrm{d} \rho .
$$

We can find $\xi_{1}>\xi_{0}$ such that

$$
\int_{c_{j}-h_{j}}^{c_{j}+h_{j}} k_{j}(\rho) T\left(\rho, \xi_{0}\right) \mathrm{d} \rho=\theta_{4} F_{j}, \int_{c_{j}-h_{j}}^{c_{j}+h_{j}} k_{j}(\rho) T\left(\rho, \xi_{1}\right) \mathrm{d} \rho=\theta_{1} F_{j},
$$

and for every $\xi \in\left[\xi_{0}, \xi_{1}\right]$ we have

$$
\theta_{1} F_{j} \leqslant \int_{c_{j}-h_{j}}^{c_{j}+h_{j}} k_{j}(\rho) T(\rho, \xi) \mathrm{d} \rho \leqslant \theta_{4} F_{j} .
$$

Let us denote by $\tilde{D}_{j}^{+}$the region bounded by the curves $\rho=c_{j} \pm h_{j}$ and $\xi=\xi_{0}, \xi_{1}$. This region depends on time, but we will suppress this dependence in notation. Theorem 4 will follow from the following

THEOREM 6. - Let $T(x, y, t)$ be a solution of (3.1) with the boundary conditions (1.3) and (1.4) or (1.5), with the initial data $T_{0}(x, y)$ satisfying (3.3), and nonlinearity $f(T)$ satisfying (1.8). Then under the assumptions (3.4) and (3.6) on the streamlines of the flow $u(x, y) \in C^{1}(\Omega)$, we have for every time $t$

$$
\int_{\tilde{D}_{j}^{+}}\left(T_{t}+\kappa|\nabla T|^{2}+\frac{v_{0}^{2}}{\kappa} f(T)\right) \mathrm{d} x \mathrm{~d} y \geqslant C\left(\zeta, f_{0}\right)\left(1+\frac{l}{h}\right)^{-1}\left(\theta_{4}-\theta_{1}\right)^{3} F_{j}
$$

with the constants $\zeta, f_{0}$ and $\theta_{1,4}$ defined by (1.11).

Remark. - A similar lower bound on the integral over $D_{j}^{+}$is easier to obtain, and also suffices to prove Theorem 4. We chose, however, to formulate Theorem 6 in this stronger version since this is what we will need when dealing with cellular flows.

Proof. - Let $\theta_{3}=\theta_{4}-\left(\theta_{4}-\theta_{1}\right) / 3$, and $\theta_{2}=\theta_{1}+\left(\theta_{4}-\theta_{1}\right) / 3$. Then we may choose $\eta_{0}$ and $\eta_{1}$ similarly to the shear case, namely

$$
\begin{aligned}
& \eta_{0}=\inf _{\eta}\left\{\eta>0: \int_{c_{j}-h_{j}}^{c_{j}+h_{j}} \mathrm{~d} \rho k_{j}(\rho) T\left(\rho, \xi_{0}+\eta\right)=\theta_{3} F_{j}\right\}, \\
& \eta_{1}=\inf _{\eta}\left\{\eta>0: \int_{c_{j}-h_{j}}^{c_{j}+h_{j}} \mathrm{~d} \rho k_{j}(\rho) T\left(\rho, \xi_{1}-\eta\right)=\theta_{2} F_{j}\right\} .
\end{aligned}
$$


We choose $\alpha \in\left(0, \eta_{0}\right)$ and $\beta \in\left(0, \eta_{1}\right)$, integrate (3.1) over $\xi \in\left(\eta_{0}+\alpha, \eta_{1}-\beta\right)$ and average in $\rho$ with the kernel $G$ to get

$$
\begin{aligned}
& \int_{\xi_{0}+\alpha}^{\xi_{1}-\beta} \mathrm{d} \xi \int_{c_{j}-h_{j}}^{c_{j}+h_{j}} \mathrm{~d} \rho G\left(h_{j}, \rho-c_{j}\right) E_{1} E_{2} T_{t} \\
& \quad-\kappa \int_{\xi_{0}+\alpha}^{\xi_{1}-\beta} \mathrm{d} \xi \int_{c_{j}-h_{j}}^{c_{j}+h_{j}} \mathrm{~d} \rho G\left(h_{j}, \rho-c_{j}\right) E_{1} E_{2}\left[T_{x x}+T_{y y}\right] \\
& \geqslant \int_{c_{j}-h_{j}}^{c_{j}+h_{j}} k_{j}(\rho)\left[T\left(\rho, \xi_{0}+\alpha\right)-T\left(\rho, \xi_{1}-\beta\right)\right] \mathrm{d} \rho \\
& \geqslant \frac{1}{3}\left(\theta_{4}-\theta_{1}\right) F_{j} .
\end{aligned}
$$

In (3.10), we dropped the term involving $f(T)$ on the right-hand side, as we did in the shear case. We now look at the term involving the Laplacian:

$$
\begin{aligned}
& \int_{\xi_{0}+\alpha}^{\xi_{1}-\beta} \mathrm{d} \xi \int_{c_{j}-h_{j}}^{c_{j}+h_{j}} \mathrm{~d} \rho G\left(h_{j}, \rho-c_{j}\right) E_{1}(\rho, \xi) E_{2}(\rho, \xi)\left[T_{x x}(\rho, \xi)+T_{y y}(\rho, \xi)\right] \\
& =\frac{1}{h_{j}} \int_{0}^{h_{j}} \mathrm{~d} \delta \int_{\xi_{0}+\alpha}^{\xi_{1}-\beta} \mathrm{d} \xi \int_{c_{j}-\delta}^{c_{j}+\delta} \mathrm{d} \rho E_{1}(\rho, \xi) E_{2}(\rho, \xi)\left[T_{x x}(\rho, \xi)+T_{y y}(\rho, \xi)\right] .
\end{aligned}
$$

Let us denote $D_{\delta \alpha \beta}$ the region bounded by coordinate curves $\rho=c_{j} \pm \delta$ and $\xi=\xi_{0}+$ $\alpha, \xi=\xi_{1}-\beta$. Using Green's formula, we rewrite the last two integrations for fixed $\delta$ as

$$
\begin{aligned}
& \int_{\xi_{0}+\alpha}^{\xi_{1}-\beta} \mathrm{d} \xi \int_{c_{j}-\delta}^{c_{j}+\delta} \mathrm{d} \rho E_{1}(\rho, \xi) E_{2}(\rho, \xi)\left[T_{x x}(\rho, \xi)+T_{y y}(\rho, \xi)\right] \\
& =\iint_{D_{\delta \alpha \beta}} \Delta T \mathrm{~d} x \mathrm{~d} y=\int_{\partial D_{\delta \alpha \beta}} \frac{\partial T}{\partial n} \mathrm{~d} s \\
& =\int_{\xi_{0}+\alpha}^{\xi_{1}-\beta} \mathrm{d} \xi\left[\omega\left(c_{j}+\delta, \xi\right) \frac{\partial T}{\partial \rho}\left(c_{j}+\delta, \xi\right)-\omega\left(c_{j}-\delta, \xi\right) \frac{\partial T}{\partial \rho}\left(c_{j}-\delta, \xi\right)\right] \\
& \quad+\int_{c_{j}-\delta}^{c_{j}+\delta} \mathrm{d} \rho\left[\omega^{-1}\left(\rho, \xi_{1}-\beta\right) \frac{\partial T}{\partial \xi}\left(\rho, \xi_{1}-\beta\right)-\omega^{-1}\left(\rho, \xi_{0}+\alpha\right) \frac{\partial T}{\partial \xi}\left(\rho, \xi_{0}+\alpha\right)\right] .
\end{aligned}
$$

We used the definition (3.5) of the function $\omega(\rho, \xi)=E_{2}(\rho, \xi) / E_{1}(\rho, \xi)$ in the last step. The average of the term on the first line in (3.11) is estimated by the following lemma, an analog of Lemma 2. 
LEMmA 4. - There exists a constant $C>0$ such that we have for all $\xi \in\left[\xi_{0}, \xi_{1}\right]$

$$
\begin{aligned}
& \frac{\kappa}{h_{j}}\left|\int_{0}^{h_{j}} \mathrm{~d} \delta\left[\omega\left(c_{j}+\delta, \xi\right) \frac{\partial T}{\partial \rho}\left(c_{j}+\delta, \xi\right)-\omega\left(c_{j}-\delta, \xi\right) \frac{\partial T}{\partial \rho}\left(c_{j}-\delta, \xi\right)\right]\right| \\
& \quad \leqslant C f_{0}^{-1 / 2} \zeta^{-1} \frac{\kappa}{v_{0} h_{j}}\left[\frac{v_{0}^{2}}{\kappa} \int_{c_{j}-h_{j}}^{c_{j}+h_{j}} \mathrm{~d} \rho E_{1} E_{2} f(T)+\kappa \int_{c_{j}-h_{j}}^{c_{j}+h_{j}} \mathrm{~d} \rho E_{1} E_{2}|\nabla T|^{2}\right],
\end{aligned}
$$

where the constant $C$ depends only on constants in the bounds (3.6) and (3.4).

Proof. - We will show that

$$
\begin{aligned}
& \left|\int_{0}^{h_{j}} \mathrm{~d} \delta \omega\left(c_{j}+\delta, \xi\right) \frac{\partial T}{\partial \rho}\left(c_{j}+\delta, \xi\right)\right| \\
& \leqslant \frac{C f_{0}^{-1 / 2} \zeta^{-1}}{v_{0}}\left[\kappa \int_{c_{j}}^{c_{j}+h_{j}} \mathrm{~d} \rho T_{\rho}^{2}(\rho, \xi)+\frac{v_{0}^{2}}{\kappa} \int_{c_{j}}^{c_{j}+h_{j}} \mathrm{~d} \rho f(T(\rho, \xi))\right]
\end{aligned}
$$

for all $\xi \in\left[\xi_{0}, \xi_{1}\right]$. A similar estimate holds for the second term on the left side of (3.12). By definition of $\xi_{0}$ and $\xi_{1}$ for every $\xi \in\left[\xi_{0}, \xi_{1}\right]$ there exists $\rho_{0} \in\left(c_{j}-h_{j}, c_{j}+h_{j}\right)$ such that $T\left(\rho_{0}, \xi\right) \in\left[\theta_{1}, \theta_{4}\right]$. Then given $\xi \in\left[\xi_{0}, \xi_{1}\right]$ we have two possibilities. First, assume that $T \in\left(\theta_{1}-\zeta, \theta_{4}+\zeta\right)$ for all $\rho \in\left(c_{j}-h_{j}, c_{j}+h_{j}\right)$. Then we have for such $\xi$

$$
\int_{c_{j}}^{c_{j}+h_{j}} \mathrm{~d} \rho f(T(\rho, \xi)) \geqslant C f_{0} \int_{c_{j}}^{c_{j}+h_{j}} \mathrm{~d} \rho \omega^{2}(\rho, \xi),
$$

which implies that

$$
\begin{aligned}
& \left|\int_{0}^{h_{j}} \mathrm{~d} \delta \omega\left(c_{j}+\delta, \xi\right) \frac{\partial T}{\partial \rho}\left(c_{j}+\delta, \xi\right)\right| \\
& \quad \leqslant\left(\int_{c_{j}}^{c_{j}+h_{j}} \mathrm{~d} \rho \omega^{2}(\rho, \xi)\right)^{1 / 2}\left(\int_{c_{j}}^{c_{j}+h_{j}} \mathrm{~d} \rho T_{\rho}^{2}(\rho, \xi)\right)^{1 / 2} \\
& \quad \leqslant C f_{0}^{-1 / 2}\left(\int_{c_{j}}^{c_{j}+h_{j}} \mathrm{~d} \rho f(T(\rho, \xi))\right)^{1 / 2}\left(\int_{c_{j}}^{c_{j}+h_{j}} \mathrm{~d} \rho\left(T_{\rho}^{2}(\rho, \xi)\right)^{1 / 2}\right. \\
& \quad \leqslant \frac{C f_{0}^{-1 / 2}}{v_{0}}\left[\kappa \int_{c_{j}}^{c_{j}+h_{j}} \mathrm{~d} \rho T_{\rho}^{2}(\rho, \xi)+\frac{v_{0}^{2}}{\kappa} \int_{c_{j}}^{c_{j}+h_{j}} \mathrm{~d} \rho f(T(\rho, \xi))\right] .
\end{aligned}
$$

The other case for a given $\xi$ is that the temperature $T(\rho, \xi)$ drops out of the range $\left(\theta_{1}-\zeta, \theta_{4}+\zeta\right)$ for some $\rho$. Then we may find $\rho^{\prime} \in\left(c_{j}-h, c_{j}+h_{j}\right)$ such that 
$T\left(\rho^{\prime}, \xi\right)=\theta_{1}-\zeta$ or $T\left(\rho^{\prime}, \xi\right)=\theta_{4}+\zeta$, and, moreover, $T \in\left(\theta_{1}-\zeta, \theta_{4}+\zeta\right)$ for all $\rho$ between $\rho^{\prime}$ and $\rho_{0}$. Then we have $\left|T\left(\rho^{\prime}, \xi\right)-T\left(\rho_{0}, \xi\right)\right| \geqslant \zeta$ and hence

$$
\begin{aligned}
& \left(\int_{c_{j}}^{c_{j}+h_{j}} \mathrm{~d} \rho f(T(\rho, \xi))\right)^{1 / 2}\left(\int_{c_{j}}^{c_{j}+h_{j}} \mathrm{~d} \rho T_{\rho}^{2}(\rho, \xi)\right)^{1 / 2} \\
& \geqslant\left(\frac{\zeta^{2}}{\left|\rho^{\prime}-\rho_{0}\right|} f_{0}\left|\rho^{\prime}-\rho_{0}\right|\right)^{1 / 2} \geqslant \zeta f_{0}^{1 / 2} .
\end{aligned}
$$

Then the estimate (3.13) also holds in that case since

$$
\left|\int_{0}^{h_{j}} \mathrm{~d} \delta \omega\left(c_{j}+\delta, \xi\right) \frac{\partial T}{\partial \rho}\left(c_{j}+\delta, \xi\right)\right| \leqslant C
$$

for all $\xi$, as can be seen from integrating by parts in $\rho$ and using (3.6). Since $\zeta \leqslant 1 / 2$ (3.13) holds in both cases.

In order to bring the term in the second line of (3.11) into a form convenient for analysis we average (3.11) in $\alpha$ and $\beta$. Let us consider an estimate on the second summand after averaging in $\alpha$ over $\left[0, \eta_{0}\right]$; the other summand is treated similarly.

LEMMA 5. - There exists a constant $C$, depending only on constants in bounds (3.4) and (3.6), such that

$$
\begin{gathered}
\left|\frac{\kappa}{\eta_{0} h_{j}} \int_{0}^{h_{j}} \mathrm{~d} \delta \int_{0}^{\eta_{0}} \mathrm{~d} \alpha \int_{c_{j}-\delta}^{c_{j}+\delta} \mathrm{d} \rho \omega^{-1}\left(\rho, \xi_{0}+\alpha\right) \frac{\partial T}{\partial \xi}\left(\rho, \xi_{0}+\alpha\right)\right| \\
\leqslant C\left[f_{0}^{-1 / 2}\left(\theta_{4}-\theta_{1}\right)^{-1} \min \left(\left(\theta_{4}-\theta_{1}\right), \zeta\right)^{-1} \frac{\kappa}{v_{0} h_{j}}\right. \\
\quad \times \int_{\tilde{D}_{j}^{+}} \mathrm{d} x \mathrm{~d} y\left[\frac{v_{0}^{2}}{\kappa} f(T(x, y))+\kappa|\nabla T(x, y)|^{2}\right] \\
\left.+\left(\theta_{4}-\theta_{1}\right)^{-2} \kappa \int_{\tilde{D}_{j}^{+}} \mathrm{d} x \mathrm{~d} y|\nabla T(x, y)|^{2}\right] .
\end{gathered}
$$

Proof. - We have to estimate the following expression

$$
\begin{aligned}
& \frac{1}{\eta_{0}} \int_{0}^{h_{j}} \mathrm{~d} \delta \int_{0}^{\eta_{0}} \mathrm{~d} \alpha \int_{c_{j}-\delta}^{c_{j}+\delta} \mathrm{d} \rho \omega^{-1}\left(\rho, \xi_{0}+\alpha\right) \frac{\partial T}{\partial \xi}\left(\rho, \xi_{0}+\alpha\right) \\
& \quad=\frac{1}{\eta_{0}} \int_{0}^{h_{j}} \mathrm{~d} \delta \int_{c_{j}-\delta}^{c_{j}+\delta} \mathrm{d} \rho\left[\omega^{-1}\left(\rho, \xi_{0}+\eta_{0}\right) T\left(\rho, \xi_{0}+\eta_{0}\right)-\omega^{-1}\left(\rho, \xi_{0}\right) T\left(\rho, \xi_{0}\right)\right]
\end{aligned}
$$




$$
-\frac{1}{\eta_{0}} \int_{0}^{h_{j}} \mathrm{~d} \delta \int_{0}^{\eta_{0}} \mathrm{~d} \alpha \int_{c_{j}-\delta}^{c_{j}+\delta} \mathrm{d} \rho \frac{\partial \omega^{-1}}{\partial \xi}\left(\rho, \xi_{0}+\alpha\right) T\left(\rho, \xi_{0}+\alpha\right) .
$$

The first term in (3.15) is evidently bounded by

$$
\frac{1}{\eta_{0}}\left|\int_{0}^{h_{j}} \mathrm{~d} \delta \int_{c_{j}-\delta}^{c_{j}+\delta} \mathrm{d} \rho\left[\omega^{-1}\left(\rho, \xi_{0}+\eta_{0}\right) T\left(\rho, \xi_{0}+\eta_{0}\right)-\omega^{-1}\left(\rho, \xi_{0}\right) T\left(\rho, \xi_{0}\right)\right]\right| \leqslant \frac{C h_{j}^{2}}{\eta_{0}} .
$$

Furthermore, we claim that

$$
\int_{\tilde{D}_{j}^{+}} \mathrm{d} x \mathrm{~d} y|\nabla T(x, y)|^{2} \geqslant \frac{C\left(\theta_{4}-\theta_{3}\right)^{2} h_{j}}{\eta_{0}} .
$$

This is shown exactly as the estimate (2.22) in the proof of Lemma 3 for the shear case, given the assumption (3.6) on $\omega$. We combine (3.16) and (3.17) to obtain

$$
\begin{aligned}
& \frac{1}{\eta_{0}}\left|\int_{0}^{h_{j}} \mathrm{~d} \delta \int_{c_{j}-\delta}^{c_{j}+\delta} \mathrm{d} \rho\left[\omega^{-1}\left(\rho, \xi_{0}+\eta_{0}\right) T\left(\rho, \xi_{0}+\eta_{0}\right)-\omega^{-1}\left(\rho, \xi_{0}\right) T\left(\rho, \xi_{0}\right)\right]\right| \\
& \quad \leqslant C\left(\theta_{4}-\theta_{3}\right)^{-2} h_{j} \int_{\tilde{D}_{j}^{+}} \mathrm{d} x \mathrm{~d} y|\nabla T(x, y)|^{2} .
\end{aligned}
$$

Next we estimate the second term in (3.15):

$$
\frac{1}{\eta_{0}}\left|\int_{0}^{h_{j}} \mathrm{~d} \delta \int_{\xi_{0}}^{\xi_{0}+\eta_{0}} \mathrm{~d} \xi \int_{c_{j}-\delta}^{c_{j}+\delta} \mathrm{d} \rho \frac{\partial \omega^{-1}}{\partial \xi}(\rho, \xi) T(\rho, \xi)\right| \leqslant C h_{j}
$$

because of (3.6). Notice that if $\eta_{0} \leqslant h_{j}$, then Lemma 5 follows directly from (3.17). Hence we can assume $\eta_{0}>h_{j}$. The following final lemma allows us to finish the proof of Lemma 5.

Lemma 6. - Assume that $\eta_{0}>h_{j}$. Then there exists a constant $C$, which depends only on the constants in bounds (3.6) and (3.4), such that

$$
\int_{\tilde{D}_{j}^{+}} f(T) \mathrm{d} x \mathrm{~d} y \int_{\tilde{D}_{j}^{+}}|\nabla T|^{2} \mathrm{~d} x \mathrm{~d} y \geqslant C f_{0}\left(\theta_{4}-\theta_{1}\right)^{2}\left(\min \left(\left(\theta_{4}-\theta_{1}\right), \zeta / 2\right)\right)^{2} h_{j}^{2} .
$$

Remark. - This lemma is much easier to prove with $D_{j}^{+}$instead of $\tilde{D}_{j}^{+}$, basically the argument of Proposition 1 applies. The simpler version is also sufficient for the proof of Theorem 4, but we need this stronger version for the proof of Theorem 6 . 
Proof. - Let us denote $|S|$ the Lebesgue measure of a measurable set $S$. We know that

$$
\int_{c_{j}-h_{j}}^{c_{j}+h_{j}} k_{j}(\rho)\left(T\left(\rho, \xi_{0}\right)-T\left(\rho, \xi_{1}\right)\right) \mathrm{d} \rho=\left(\theta_{4}-\theta_{1}\right) F_{j} .
$$

Consider two sets

$$
S_{1}=\left\{\rho \mid T\left(\rho, \xi_{0}\right)-T\left(\rho, \xi_{1}\right)<\left(\theta_{4}-\theta_{1}\right) / 2\right\}, \quad S_{2}=\left[c_{j}-h_{j}, c_{j}+h_{j}\right] \backslash S_{1} .
$$

Then

$$
\int_{S_{1}} k_{j}(\rho)\left(T\left(\rho, \xi_{0}\right)-T\left(\rho, \xi_{1}\right)\right) \mathrm{d} \rho \leqslant\left(\theta_{4}-\theta_{1}\right) F_{j} / 2,
$$

and hence

$$
\int_{S_{2}} k_{j}(\rho)\left(T\left(\rho, \xi_{0}\right)-T\left(\rho, \xi_{1}\right)\right) \mathrm{d} \rho \geqslant\left(\theta_{4}-\theta_{1}\right) F_{j} / 2 .
$$

Since $T\left(\rho, \xi_{0}\right)-T\left(\rho, \xi_{1}\right) \leqslant 1$, and $k_{j}(\rho) / F_{j} \leqslant 2 h_{j}^{-1}$ by the properties of $G$ and (3.7) we must have $\left|S_{2}\right| \geqslant\left(\theta_{4}-\theta_{1}\right) h_{j} / 4$. Choose $\rho_{0} \in S_{2}$ such that

$$
\int_{\xi_{0}}^{\xi_{1}} \mathrm{~d} \xi f\left(T\left(\rho_{0}, \xi\right)\right) \leqslant \frac{3}{\left|S_{2}\right|} \int_{\tilde{D}_{j}^{+}} f(T) \mathrm{d} x \mathrm{~d} y
$$

and

$$
\int_{\xi_{0}}^{\xi_{1}} \mathrm{~d} \xi T_{\xi}^{2}\left(\rho_{0}, \xi\right) \leqslant \frac{3}{\left|S_{2}\right|} \int_{\tilde{D}_{j}^{+}} T_{\xi}^{2} \mathrm{~d} \rho \mathrm{d} \xi \leqslant \frac{C}{\left|S_{2}\right|} \int_{\tilde{D}_{j}^{+}}|\nabla T|^{2} \mathrm{~d} x \mathrm{~d} y,
$$

with $C$ depending only on the constants in (3.6). Since $\rho_{0} \in S_{2}$, we have $T\left(\rho_{0}, \xi_{0}\right)-$ $T\left(\rho_{0}, \xi_{1}\right) \geqslant\left(\theta_{4}-\theta_{1}\right) / 2$. Assume first that there exists $\xi \in\left[\xi_{0}, \xi_{1}\right]$ where $T\left(\rho_{0}, \xi\right) \in$ $\left[\theta_{1}-\zeta / 2, \theta_{4}+\zeta / 2\right]$. Then we can find $\xi_{0} \leqslant \xi_{2} \leqslant \xi_{3} \leqslant \xi_{1}$ such that for every $\xi \in\left[\xi_{2}, \xi_{3}\right]$, $T\left(\rho_{0}, \xi\right) \in\left[\theta_{1}-\zeta, \theta_{4}+\zeta\right]$, and

$$
T\left(\rho_{0}, \xi_{2}\right)-T\left(\rho_{0}, \xi_{3}\right) \geqslant \min \left(\left(\theta_{4}-\theta_{1}\right) / 2, \zeta / 2\right) .
$$

Therefore,

$$
\int_{\xi_{0}}^{\xi_{1}} f(T) \mathrm{d} \xi \geqslant f_{0}\left(\xi_{3}-\xi_{2}\right)
$$

while

$$
\int_{\xi_{0}}^{\xi_{1}} T_{\xi}^{2} \mathrm{~d} \xi \geqslant \frac{\left(\min \left(\left(\theta_{4}-\theta_{1}\right) / 2, \zeta / 2\right)\right)^{2}}{\xi_{3}-\xi_{2}}
$$


Combining these estimates and the definition of $\rho_{0}$, we find

$$
\int_{\tilde{D}_{j}^{+}} f(T) \mathrm{d} x \mathrm{~d} y \int_{\tilde{D}_{j}^{+}}|\nabla T|^{2} \mathrm{~d} x \mathrm{~d} y \geqslant C f_{0}\left(\min \left(\left(\theta_{4}-\theta_{1}\right) / 2, \zeta / 2\right)\right)^{2}\left|S_{2}\right|^{2},
$$

which proves the lemma in this case.

The other case we have to consider is that for every $\xi \in\left[\xi_{0}, \xi_{1}\right], T\left(\rho_{0}, \xi\right) \notin\left[\theta_{1}-\right.$ $\left.\zeta / 2, \theta_{4}+\zeta / 2\right]$. Then we will be able to find a point $\xi \in\left[\xi_{0}, \xi_{1}\right]$ so that there is a drop in temperature along the curve $\xi=$ const. Assume $T\left(\rho_{0}, \xi\right)>\theta_{4}+\zeta / 2$ for all $\xi$, the other case being similar. By mean value theorem, we also have that for every $\xi \in\left[\xi_{0}, \xi_{1}\right]$ there exists $\rho_{1}$ such that $T\left(\rho_{1}, \xi\right) \in\left[\theta_{1}, \theta_{4}\right]$, and so $T\left(\rho_{1}, \xi\right) \leqslant \theta_{4}$. Similarly to the above, we can find $\tilde{\xi}$ such that

$$
\int_{c_{j}-h_{j}}^{c_{j}+h_{j}} \mathrm{~d} \rho f(T(\rho, \tilde{\xi})) \leqslant \frac{3}{\xi_{1}-\xi_{0}} \int_{\tilde{D}_{j}^{+}} f(T) \mathrm{d} x \mathrm{~d} y \leqslant \frac{3}{\eta_{0}} \int_{\tilde{D}_{j}^{+}} f(T) \mathrm{d} x \mathrm{~d} y
$$

and

$$
\int_{c_{j}-h_{j}}^{c_{j}+h_{j}} \mathrm{~d} \rho T_{\rho}^{2}(\rho, \tilde{\xi}) \leqslant \frac{3}{\xi_{1}-\xi_{0}} \int_{\tilde{D}_{j}^{+}} T_{\rho}^{2} \mathrm{~d} \rho \mathrm{d} \xi \leqslant \frac{3}{\eta_{0}} \int_{\tilde{D}_{j}^{+}} T_{\rho}^{2} \mathrm{~d} \rho \mathrm{d} \xi \leqslant \frac{C}{\eta_{0}} \int_{\tilde{D}_{j}^{+}}|\nabla T|^{2} \mathrm{~d} x \mathrm{~d} y .
$$

An argument identical to the one we used in the previous case establishes that

$$
\int_{c_{j}-h_{j}}^{c_{j}+h_{j}} f(T) \mathrm{d} \rho \geqslant f_{0}\left(\rho_{1}-\rho_{2}\right)
$$

while

$$
\int_{c_{j}-h_{j}}^{c_{j}+h_{j}} T_{\rho}^{2} \mathrm{~d} \rho \geqslant \frac{\zeta^{2}}{4\left(\rho_{1}-\rho_{2}\right)}
$$

for some $c_{j}+h_{j} \geqslant \rho_{1}>\rho_{2} \geqslant c_{j}-h_{j}$. This implies that

$$
\int_{\tilde{D}_{j}^{+}} f(T) \mathrm{d} x \mathrm{~d} y \int_{\tilde{D}_{j}^{+}}|\nabla T|^{2} \mathrm{~d} x \mathrm{~d} y \geqslant C f_{0} \zeta^{2} \eta_{0}^{2} \geqslant C f_{0} \zeta^{2} h_{j}^{2} .
$$

Thus Lemma 6 is proven.

Lemma 6 and the assumptions (3.4) and (3.6) imply that

$$
\int_{\tilde{D}_{j}^{+}} \mathrm{d} x \mathrm{~d} y\left[\frac{v_{0}}{\kappa} f(T)+\frac{\kappa}{v_{0}}|\nabla T|^{2}\right] \geqslant C f_{0}^{1 / 2}\left(\theta_{4}-\theta_{1}\right) \min \left(\left(\theta_{4}-\theta_{1}\right) / 2, \zeta / 2\right) h_{j} .
$$


Therefore we deduce from (3.19) that

$$
\begin{aligned}
& \frac{1}{\eta_{0} h_{j}}\left|\int_{0}^{h_{j}} \mathrm{~d} \delta \int_{\xi_{0}}^{\xi_{0}+\eta_{0}} \mathrm{~d} \xi \int_{c_{j}-\delta}^{c_{j}+\delta} \mathrm{d} \rho \frac{\partial \omega^{-1}}{\partial \xi}(\rho, \xi) T(\rho, \xi)\right| \\
& \leqslant C f_{0}^{-1 / 2}\left(\theta_{4}-\theta_{1}\right)^{-1} \min \left(\left(\theta_{4}-\theta_{1}\right) / 2, \zeta / 2\right)^{-1} \frac{1}{v_{0} h_{j}} \\
& \quad \times \int_{\tilde{D}_{j}^{+}} \mathrm{d} x \mathrm{~d} y\left[\frac{v_{0}^{2}}{\kappa} f(T)+\kappa|\nabla T|^{2}\right] .
\end{aligned}
$$

Then (3.15), (3.18) and (3.20) imply Lemma 5.

Averaging of (3.11) in $\beta \in\left[0, b_{1}\right]$ produces terms similar to the left side of (3.14), which are bounded by the same quantity. Now we put together our estimates on $\tilde{D}_{j}^{+}$, that is, Eqs. (3.10) and (3.11), and Lemmas 4 and 5:

$$
\begin{aligned}
& \int_{\tilde{D}_{j}^{+}} \mathrm{d} x \mathrm{~d} y G\left(h_{j}, \rho-c_{j}\right) T_{t}+\zeta^{-1} f_{0}^{-1 / 2} \frac{\kappa}{v_{0} h_{j}} \int_{\tilde{D}_{j}^{+}} \mathrm{d} x \mathrm{~d} y\left[\frac{v_{0}^{2}}{\kappa} f(T)+\kappa|\nabla T|^{2}\right] \\
& +C f_{0}^{-1 / 2}\left(\theta_{4}-\theta_{1}\right)^{-1} \min \left(\left(\theta_{4}-\theta_{1}\right), \zeta\right)^{-1} \frac{\kappa}{v_{0} h_{j}} \int_{\tilde{D}_{j}^{+}} \mathrm{d} x \mathrm{~d} y\left[\frac{v_{0}^{2}}{\kappa} f(T)+\kappa|\nabla T|^{2}\right] \\
& +C\left(\theta_{4}-\theta_{1}\right)^{-2} \kappa \int_{\tilde{D}_{j}^{+}} \mathrm{d} x \mathrm{~d} y|\nabla T|^{2} \geqslant C\left(\theta_{4}-\theta_{1}\right) F_{j} .
\end{aligned}
$$

Therefore we obtain

$$
\begin{gathered}
\left(1+\frac{\kappa}{v_{0} h_{j}}\right) \int_{\tilde{D}_{j}^{+}} \mathrm{d} x \mathrm{~d} y\left[T_{t}+\frac{v_{0}^{2}}{\kappa} f(T)+\kappa|\nabla T|^{2}\right] \geqslant C\left(\zeta, f_{0}\right)\left(\theta_{4}-\theta_{1}\right)^{3} F_{j} \\
\geqslant C\left(\zeta, f_{0}\right)\left(\theta_{4}-\theta_{1}\right)^{3} \int_{c_{j}-h_{j}}^{c_{j}+h_{j}} E_{1}(\rho, \xi)|u(\rho, \xi)| \mathrm{d} \xi
\end{gathered}
$$

which proves Theorem 6 .

The estimate on the regions $D_{j}^{-}$with $u \cdot \nabla \xi<0$ is similar. The only essential difference is that in inequality (3.10) we drop the term involving $T_{t}$ but keep the one with $f(T)$, which does not make any difference in the final result. Then Theorem 4 follows after summation over all $D_{j}^{ \pm}$from Lemma 1 and Proposition 1 .

\section{Cellular flows: The main result}

Now we consider (1.2) in a cellular flow:

$$
T_{t}+u \cdot \nabla T=\kappa \Delta T+\frac{v_{0}^{2}}{\kappa} f(T),
$$


that is, flow with closed streamlines, and establish a lower bound for the burning rate. For simplicity, we limit our consideration to one typical representative class of cellular flows, given by the stream function

$$
\psi(x, y)=U H \sin \frac{x}{H} \sin \frac{y}{H}
$$

on the strip $(-\infty, \infty) \times[0, \pi H]$ (for convenience from now on $H$ will be the width of the strip divided by $\pi)$. The flow $u(x, y)$ is given by

$$
u(x, y)=U \nabla^{\perp} \psi(x, y)=U\left(\sin \frac{x}{H} \cos \frac{y}{H},-\cos \frac{x}{H} \sin \frac{y}{H}\right) .
$$

The streamlines inside a period cell are depicted on Fig. 2. The results we prove can be extended in a direct way to the periodic cellular flows of more general form.

We will further assume that the Peclet number is larger than one:

$$
\mathrm{Pe}=\frac{U H}{\kappa} \geqslant 1
$$

and the size of the cell is larger than the laminar front width:

$$
\frac{l}{H} \leqslant 1, \quad l=\frac{\kappa}{v_{0}} .
$$

Conditions (4.4) and (4.5) are natural for flows of large amplitude and for thin fronts. Moreover, our results may be easily adapted to the other regimes, where (4.4) and (4.5) are violated. We introduce also the turnover time $\tau_{u}$, and the chemical reaction time $\tau_{c}$ :

$$
\tau_{u}=\frac{H}{U}, \quad \tau_{c}=\frac{\kappa}{v_{0}^{2}} .
$$

It turns out that the ratio $\tau_{u} / \tau_{c}$ is the crucial parameter for burning in the cellular flows.

Finally we assume that $T(x, y, t)$ satisfies the usual boundary conditions and that

$$
\frac{\partial T}{\partial t}(x, y, t) \geqslant 0 .
$$

As we noted previously, this condition is satisfied as long as it holds initially.

THEOREM 7. - Let $T(x, y, t)$ be a solution of (2.1) with the boundary conditions (1.3) and either (1.4) or (1.5), and the cellular flow given by (4.3). Let the initial data $T_{0}(x, y)$ satisfy (2.4), (1.6) and (1.7), and let the non-linearity $f(T)$ be of either ignition or general KPP type. Furthermore, assume that (4.4) and (4.5) hold. Then we have for any time $t$

$$
V(t) \geqslant \begin{cases}\left(C_{1} \sqrt{\frac{\tau_{c}}{\tau_{u}}}+C_{2}\right) v_{0}, & \text { if } \tau_{c} \leqslant \tau_{u}, \\ \left(C_{1}\left(\frac{\tau_{c}}{\tau_{u}}\right)^{1 / 5}+C_{2}\right) v_{0}, & \text { if } \tau_{c} \geqslant \tau_{u} .\end{cases}
$$


The constants in the inequalities depend only on the reaction $f$, more particularly on constants $f_{0}, \zeta$, and $\theta_{4}-\theta_{1}$ that appear in (1.11).

Remark. - As we noted above, in order to avoid excessive details, we chose not to formulate Theorem 7 in the exhaustive form which goes through all possible relationships between parameters (large $\kappa$ limit, small $H$ limit, small $v_{0}$ limit). The reader will find it not difficult to extend the results we prove to the above mentioned regimes. Theorem 7 is formulated here for the range of parameters that appears to be physically reasonable for most problems of interest.

Furthermore, we have the following corollary.

COROLLARY 3. - Let $f(T)$ be of ignition nonlinearity type (1.10), or of the KPP type (1.9) and let $c$ be the speed of a traveling wave-type solution $T(x, y, t)=U(x-c t, x, y)$ of (4.1), periodic in the second two variables. Then there exist constants $C_{1,2}>0$ which depend only on the function $f$ and on the constants appearing in (3.4) and (3.6) such that

$$
c \geqslant \begin{cases}\left(C_{1} \sqrt{\frac{\tau_{c}}{\tau_{u}}}+C_{2}\right) v_{0}, & \text { if } \tau_{c} \leqslant \tau_{u}, \\ \left(C_{1}\left(\frac{\tau_{c}}{\tau_{u}}\right)^{1 / 5}+C_{2}\right) v_{0}, & \text { if } \tau_{c} \geqslant \tau_{u} .\end{cases}
$$

Corollary 3 follows from Theorem 7 since the traveling front profile $U(s, x, y)$ is monotonically decreasing in $s$ (see [31] for the ignition case, and [3] for KPP). Then Theorem 2 follows immediately from Corollary 3, results of [31] and the argument we gave in the proof of Theorem 1.

The proof of Theorem 7 is a boundary layer argument that proceeds, roughly, as follows. The temperature drops from one on the left to zero on the right. We will watch the temperature in the layers of width $h$ formed by streamlines near the boundary of the cells. The drop of temperature in these layers may occur inside the cells or over the diffusive interfaces. The first estimate, which we call advective, shows how much the cell must contribute to the bulk burning rate if a certain drop of the temperature (in the range $\left[\theta_{1}, \theta_{4}\right]$ ) takes place along the streamlines inside the cell. It is reasonable to expect that the drop over the cell will be small when advection is strong since it mixes the fluid inside the cell quickly: in an analytic form this intuition will translate into a large lower bound for the burning rate if the temperature drop is significant. The second estimate, which we call diffusive, gives a lower bound for the burning rate given certain drop of the temperature between the two cells. We do expect the temperature to drop on the boundaries, and hence the lower bound is only effective if we choose $h$ in an appropriate way, sufficiently small. Finally, we prove the reaction estimate, which takes into account the total area of the region over which the temperature drops. These estimates will be brought together to establish the lower bound for the bulk burning rate using an appropriate optimization argument.

\section{Cellular flows: regularity of the streamlines}

Our first objective is to define appropriate curvilinear coordinates on the cells, and to show that these coordinates satisfy certain technical assumptions that we will need. The 
natural choice of the coordinate $\rho$, which is constant on the streamlines, is

$$
\rho(x, y)=\frac{\psi(x, y)}{U}=H \sin \frac{x}{H} \sin \frac{y}{H} .
$$

We have certain freedom in the definition of the orthogonal coordinate $\xi$ along the streamlines:

$$
\nabla \xi=Q \nabla^{\perp} \rho,
$$

where $Q$ is some function which should satisfy

$$
\nabla \rho \cdot \nabla Q=-Q \Delta \rho .
$$

It is easy to compute that

$$
\partial_{\rho}=x_{\rho} \partial_{x}+y_{\rho} \partial_{y}=\frac{\xi_{y}}{J} \partial_{x}-\frac{\xi_{x}}{J} \partial_{y},
$$

where $J=\rho_{x} \xi_{y}-\rho_{y} \xi_{x}=-Q|\nabla \rho|^{2}$. Hence

$$
\partial_{\rho}=\frac{1}{|\nabla \rho|^{2}} \nabla \rho \cdot \nabla,
$$

and so we have from (5.3)

$$
\frac{\partial Q}{\partial \rho}=-\frac{Q \Delta \rho}{|\nabla \rho|^{2}} .
$$

We will choose $Q$ so that $Q(H / 2, \xi)=1$; Eq. (5.4) then allows us to define $Q$ in the region $H>\rho>0$ :

$$
Q(\rho, \xi)=\mathrm{e}^{-\int_{H / 2}^{\rho} \frac{\Delta \rho(h, \xi)}{|\nabla \rho(h, \xi)|^{2}} \mathrm{~d} h} .
$$

We have the following auxiliary

LEMMA 7. - In the region $H / 2 \geqslant \rho>0$ we have the following bound for the function $Q$ :

$$
0<\mathrm{e}^{-1} \leqslant Q(\rho, \xi) \leqslant \mathrm{e} .
$$

Proof. - Consider formula (5.5). We have

$$
\Delta \rho=-\frac{2 \pi^{2}}{H^{2}} \rho(x, y),
$$

and

$$
\begin{aligned}
|\nabla \rho|^{2} & =\left(\cos \frac{x}{H} \sin \frac{y}{H}\right)^{2}+\left(\sin \frac{x}{H} \cos \frac{y}{H}\right)^{2} \\
& =\left(\sin \frac{y}{H}\right)^{2}+\left(\sin \frac{x}{H}\right)^{2}-\frac{2 \rho^{2}}{H^{2}} \geqslant 2 \frac{\rho}{H}\left(1-\frac{\rho}{H}\right) \geqslant \frac{\rho}{H}
\end{aligned}
$$


in the region $\rho \leqslant H / 2$. Hence in the region $H / 2 \geqslant \rho>0$.

$$
\frac{|\Delta \rho|}{|\nabla \rho|^{2}} \leqslant 2 / H
$$

Therefore, by (5.5), we have $\mathrm{e}^{-1} \leqslant Q \leqslant \mathrm{e}$ in this region.

Recall our notation

$$
\mathrm{d} x^{2}+\mathrm{d} y^{2}=E_{1}^{2} \mathrm{~d} \rho^{2}+E_{2}^{2} \mathrm{~d} \xi^{2}, \quad \omega=\frac{E_{2}}{E_{1}} .
$$

The next proposition summarizes some of the properties of the coordinates $(\rho, \xi)$ in a region of interest to us.

PRoposition 2. - For the cellular flow defined by (4.3) and coordinates $\rho$, $\xi$ defined by (5.1), (5.2), and (5.5), the following bounds hold in the region $H / 2 \geqslant \rho>0$ :

$$
\begin{array}{r}
E_{1,2}(\rho, \xi) \geqslant C, \\
0<C^{-1} \geqslant \omega(\rho, \xi) \leqslant C, \\
\left|\frac{\partial \omega}{\partial \rho}(\rho, \xi)\right| \leqslant C H^{-1}, \\
\left|\frac{\partial \omega}{\partial \xi}(\rho, \xi)\right| \leqslant C H^{-1}|\log (\rho / H)| .
\end{array}
$$

Proof. - Direct computation gives that $E_{1}=1 /|\nabla \rho|, E_{2}=1 / Q|\nabla \rho|$. Then Lemma 7 and the fact that $|\nabla \rho| \leqslant 1$ imply (5.7). It follows from the definition of $\omega$ that $\omega=1 / Q$, and hence Lemma 7 implies (5.9). Next,

$$
\left|\frac{\partial \omega}{\partial \rho}\right|=\frac{1}{Q} \frac{|\Delta \rho|}{|\nabla \rho|^{2}} \leqslant \frac{2 \mathrm{e}}{H},
$$

proving (5.10). Finally,

$$
\left|\frac{\partial \omega}{\rho \xi}\right|=\frac{2}{Q H^{2}}\left|\int_{H / 2}^{\rho} h \partial_{\xi}\left(\frac{1}{|\nabla \rho|^{2}}\right) \mathrm{d} h\right| .
$$

Notice that

$$
\partial_{\xi}=x_{\xi} \partial_{x}+y_{\xi} \partial_{y}=\frac{\rho_{y}}{Q|\nabla \rho|^{2}} \partial_{x}-\frac{\rho_{x}}{Q|\nabla \rho|^{2}} \partial_{y} .
$$

A straightforward computation using (5.5) and (5.6) leads to

$$
\left|\frac{\partial \omega}{\partial \xi}\right| \leqslant \frac{C}{H}\left|\int_{H / 2}^{\rho} \frac{\mathrm{d} h}{h}\right| \leqslant C H^{-1}|\log (\rho / H)| .
$$




\section{Cellular flows: Advective estimate}

Let us introduce some notation. Within the cell, we will normalize $\xi$ by letting it be zero in the negative direction of the $x$ axis (assuming that the origin has been placed in the center of the cell). We will denote by $L$ the value of $\xi$ in the positive direction of the $x$ axis. In every cell, we will consider a tube of streamlines bounded by $\rho=h$ and $\rho=3 h ; h$ will be always assumed to be less than $H / 6$. We set

$$
k(\rho)=G(h, \rho-2 h) E_{1}(\rho, \xi)|u(\rho, \xi)| .
$$

The fact that $k(\rho)$ does not depend on $\xi$ is a direct corollary of incompressibility of the flow. Moreover, with our definition of $\rho$ for the cellular flow, we have $k(\rho)=$ $U G(h, \rho-2 h)$. We also denote

$$
F=\int_{h}^{3 h} k(\rho) \mathrm{d} \rho .
$$

As a corollary of Proposition 2, in the strip $h \leqslant \rho \leqslant 3 h$ we have all conditions on $E_{1,2}$ and $\omega$ that have been necessary for the percolating flow estimates. In particular, we have

$$
\left|\frac{\partial \omega}{\partial \xi}\right| \leqslant C H^{-1}|\log (\rho / H)| \leqslant C h^{-1},
$$

since $h \leqslant \rho \leqslant H$. We first state an estimate very similar to Theorem 6 .

THEOREM 8. - Assume that within one cell $C$, there exist two values $\xi_{0}, \xi_{1}$ such that for some $s_{0}, s_{1} \in\left[\theta_{1}, \theta_{4}\right], s_{0}>s_{1}$, we have

$$
\begin{gathered}
\int_{h}^{3 h} k(\rho) T\left(\rho, \xi_{0}\right) \mathrm{d} \rho=s_{0} F, \quad \int_{h}^{3 h} k(\rho) T\left(\rho, \xi_{1}\right) \mathrm{d} \rho=s_{1} F, \\
s_{1} F \leqslant \int_{h}^{3 h} k(\rho) T(\rho, \xi) \mathrm{d} \rho \leqslant s_{0} F \quad \text { for } \xi \in\left[\xi_{0}, \xi_{1}\right] .
\end{gathered}
$$

Let $D$ be the region bounded by the curves $\rho=h, \rho=3 h, \xi=\xi_{0}$ and $\xi=\xi_{1}$. Then

$$
\int_{D}\left(T_{t}+\kappa|\nabla T|^{2}+\frac{v_{0}^{2}}{\kappa} f(T)\right) \mathrm{d} x \mathrm{~d} y \geqslant C\left(\zeta, f_{0}\right)\left(1+\frac{l}{h}\right)^{-1}\left(s_{0}-s_{1}\right)^{3} F .
$$

The constant $C$ in (6.2) depends only on parameters $\zeta$ and $f_{0}$ of the reaction $f$, that appear in (1.11), and on the constants in the bounds of Proposition 2.

Proof. - The proof is exactly the same as for Theorem 6 for percolating flows. We need only to replace $\theta_{1}$ with $s_{1}, \theta_{4}$ with $s_{0}$, and set $c_{j}=2 h, h_{j}=h$. 
The estimate (6.2) works well if there is a significant change of the temperature along the streamlines within the cell. But it has a serious flaw if the temperature drops gradually and there is little change of temperature inside any cell. The factor $\left(s_{0}-s_{1}\right)^{3}$ on the right hand side of the estimate makes it rather inefficient. Our next goal is to derive an estimate which has linear dependence on the temperature drop when the drop is small. Our main measurement tool for the temperature within the cell will be the following average

$$
\langle T\rangle_{\xi_{0}}=\frac{1}{2 A F} \int_{\xi_{0}-A}^{\xi_{0}+A} \mathrm{~d} \xi \int_{h}^{3 h} k(\rho) T(\rho, \xi) \mathrm{d} \rho .
$$

We will normally take $A$ so that the region of averaging in $\xi$ covers about half of the width of the strip when $\xi_{0}=0$ or $\xi_{0}=L$, hence $A \approx H$. Our measure of the temperature change along the cell will be the difference between such averages for different $\xi_{0}$.

THEOREM 9. - Assume that for all $\xi$ in a given cell we have

$$
\frac{1}{F} \int_{h}^{3 h} k(\rho) T(\rho, \xi) \mathrm{d} \rho \in\left[\theta_{1}, \theta_{4}\right] .
$$

Then for any $\xi_{0}, \xi_{1}$ we have for $A \geqslant h$

$$
\begin{gathered}
\int_{\xi_{0}-A}^{\xi_{1}+A} \int_{h}^{3 h}\left(T_{t}+\kappa|\nabla T|^{2}+\frac{v_{0}^{2}}{\kappa} f(T)\right) E_{1} E_{2} \mathrm{~d} \xi \mathrm{d} \rho \\
\geqslant C\left(f_{0}, \zeta\right)\left(1+\frac{l}{h}\right)^{-1}\left|\langle T\rangle_{\xi_{0}}-\langle T\rangle_{\xi_{1}}\right| F,
\end{gathered}
$$

where $C$ depends only on $f_{0}, \zeta$ and constants in the bounds of Proposition 2.

Proof. - We will consider the case where $\langle T\rangle_{\xi_{0}}>\langle T\rangle_{\xi_{1}}$, the other case being similar. Let us integrate

$$
T_{t}+u \cdot \nabla T-\kappa \Delta T=\frac{v_{0}^{2}}{\kappa} f(T)
$$

in $x$ and $y$ over the region where $\xi$ varies from $\xi_{2}$ to $\xi_{3}$, where $\xi_{2} \in\left[\xi_{0}-A, \xi_{0}+A\right]$, $\xi_{3} \in\left(\xi_{1}-A, \xi_{1}+A\right)$, and $\rho$ takes values between $h$ and $3 h$, with kernel $G(h, \rho-2 h)$. We obtain

$$
\begin{aligned}
\int_{\xi_{2}}^{\xi_{3}} \mathrm{~d} \xi & \int_{h}^{3 h} \mathrm{~d} \rho G(h, \rho-2 h) E_{1} E_{2} T_{t}(\rho, \xi)-\kappa \int_{\xi_{2}}^{\xi_{3}} \mathrm{~d} \xi \int_{h}^{3 h} \mathrm{~d} \rho G(h, \rho-2 h) E_{1} E_{2} \Delta T \\
\geqslant & \int_{h}^{3 h} \mathrm{~d} \rho k(\rho)\left(T\left(\rho, \xi_{2}\right)-T\left(\rho, \xi_{3}\right)\right) .
\end{aligned}
$$

As usual, our goal is to control the Laplacian term. Rewrite it in a familiar form 


$$
\begin{gathered}
\frac{\kappa}{h} \int_{0}^{h} \mathrm{~d} \delta\left\{\int_{\xi_{2}}^{\xi_{3}} \mathrm{~d} \xi\left[\frac{\partial T}{\partial \rho} \omega(\xi, 2 h+\delta)-\frac{\partial T}{\partial \rho} \omega(\xi, 2 h-\delta)\right]\right. \\
\left.+\int_{2 h-\delta}^{2 h+\delta} \mathrm{d} \rho\left[\frac{\partial T}{\partial \xi} \omega^{-1}\left(\xi_{3}, \rho\right)-\frac{\partial T}{\partial \xi} \omega^{-1}\left(\xi_{2}, \rho\right)\right]\right\} .
\end{gathered}
$$

We carry out two more averagings in (6.4)

$$
\frac{1}{4 A^{2}} \int_{\xi_{0}-A}^{\xi_{0}+A} \mathrm{~d} \xi_{2} \int_{\xi_{1}-A}^{\xi_{1}+A} \mathrm{~d} \xi_{3}
$$

to be able to estimate the second part of the Laplacian term (6.5). Let us start by estimating the first expression in square brackets, more precisely,

$$
\frac{\kappa}{h} \int_{0}^{h} \mathrm{~d} \delta \int_{\xi_{2}}^{\xi_{3}} \mathrm{~d} \xi \frac{\partial T}{\partial \rho} \omega(\xi, 2 h+\delta)
$$

(the other part is estimated similarly). Notice that by the assumption of the theorem for every $\xi$ there exists $\rho$ (depending on $\xi$ ) such that $T(\rho, \xi) \in\left[\theta_{1}, \theta_{4}\right]$. The estimate of this term now follows step by step the estimate of the same term in the proof of Theorem 6 . We get that for every $\xi \in\left[\xi_{0}-A, \xi_{1}+A\right]$,

$$
\frac{\kappa}{h} \int_{0}^{h} \mathrm{~d} \delta \frac{\partial T}{\partial \rho} \omega(\xi, 2 h+\delta) \leqslant C f^{-1 / 2} \zeta^{-1} \frac{\kappa}{v_{0} h} \int_{h}^{3 h}\left[\kappa|\nabla T|^{2}+\frac{v_{0}^{2}}{\kappa} f(T)\right] E_{1} E_{2} \mathrm{~d} \rho .
$$

The integrations in $\xi_{2,3}$ simply average out, they are not needed for this term.

Let us now consider the estimate of the second expression in square brackets in (6.5), more particularly the first summand (the second one is estimated in the same way). Averaging in $\xi_{2}$ simply disappears since there is no dependence on this variable, and we are left with

$$
\frac{\kappa}{2 h A} \int_{0}^{h} \mathrm{~d} \delta \int_{2 h-\delta}^{2 h+\delta} \mathrm{d} \rho \int_{\xi_{1}-A}^{\xi_{1}+A} \mathrm{~d} \xi_{3} \frac{\partial T}{\partial \xi} \omega^{-1}\left(\xi_{3}, \rho\right) .
$$

The following lemma is the crucial step in the proof.

LEMMA 8. - Under conditions of Theorem 9 we have

$$
\begin{aligned}
& \frac{\kappa}{2 h A} \int_{0}^{h} \mathrm{~d} \delta \int_{2 h-\delta}^{2 h+\delta} \mathrm{d} \rho \int_{\xi_{1}-A}^{\xi_{1}+A} \mathrm{~d} \xi_{3} \frac{\partial T}{\partial \xi} \omega^{-1}\left(\xi_{3}, \rho\right) \\
& \leqslant C f_{0}^{-1 / 2} \zeta^{-1} \frac{\kappa}{h v_{0}} \int_{D}\left[\kappa|\nabla T|^{2}+\frac{v_{0}^{2}}{\kappa} f(T)\right] \mathrm{d} x \mathrm{~d} y,
\end{aligned}
$$


where the constant $C$ depends only on the bounds in Proposition 2.

Proof. - Integrating by parts and using (6.1), we find that

$$
\left|\int_{\xi_{1}-A}^{\xi_{1}+A} \mathrm{~d} \xi_{3} \frac{\partial T}{\partial \xi} \omega^{-1}\left(\xi_{3}, \rho\right)\right| \leqslant C\left(1+\frac{A}{h}\right)
$$

and therefore

$$
\frac{\kappa}{2 h A}\left|\int_{0}^{h} \mathrm{~d} \delta \int_{2 h-\delta}^{2 h+\delta} \mathrm{d} \rho \int_{\xi_{1}-A}^{\xi_{1}+A} \mathrm{~d} \xi_{3} \frac{\partial T}{\partial \xi} \omega^{-1}\left(\xi_{3}, \rho\right)\right| \leqslant C \kappa\left(\frac{h}{A}+1\right) \leqslant C \kappa .
$$

We have to consider several cases.

Option 1. There exists $\rho_{0}$ such that for all $\xi \in\left[\xi_{1}-A\right.$, $\left.\xi_{1}+A\right]$, we have $T\left(\rho_{0}, \xi\right) \notin$ $\left[\theta_{1}-\zeta / 2, \theta_{4}+\zeta / 2\right]$. By mean value theorem, for every $\xi$, there also exists $\rho_{1}(\xi)$ such that $T\left(\rho_{1}, \xi\right) \in\left[\theta_{1}, \theta_{4}\right]$. Then for every $\xi$ we can find $\rho_{3}>\rho_{2}$ such that $\mid T\left(\rho_{2}, \xi\right)-$ $T\left(\rho_{3}, \xi\right) \mid=\zeta / 2$, and for every $\rho \in\left[\rho_{2}, \rho_{3}\right], T(\rho, \xi) \in\left[\theta_{1}-\zeta / 2, \theta_{4}+\zeta / 2\right]$. Therefore, as we have seen before, for every $\xi \in\left[\xi_{1}-A, \xi_{1}+A\right]$ we have

$$
\left(\int_{h}^{3 h} \mathrm{~d} \rho T_{\rho}^{2}\right)^{1 / 2}\left(\int_{h}^{3 h} \mathrm{~d} \rho f(T)\right)^{1 / 2} \geqslant \frac{\zeta f_{0}^{1 / 2}}{2} .
$$

Hence, using Cauchy-Schwartz and integrating in $\xi$, we get

$$
\int_{h}^{3 h} \mathrm{~d} \rho \int_{\xi_{1}-A}^{\xi_{1}+A} \mathrm{~d} \xi E_{1} E_{2}\left(\kappa|\nabla T|^{2}+\frac{v_{0}^{2}}{\kappa} f(T)\right) \geqslant \frac{v_{0} \zeta f_{0}^{1 / 2} A}{2} .
$$

From this inequality and (6.9) our lemma follows since $A \geqslant h$.

Option 2. For every $\rho$, there exists $\xi_{4}$ such that $T\left(\rho, \xi_{4}\right) \in\left[\theta_{1}-\zeta / 2, \theta_{4}+\zeta / 2\right]$. Here we have to consider two distinct sets of $\rho$. First, assume that $T(\rho, \xi) \in\left[\theta_{1}-\zeta, \theta_{4}+\zeta\right]$ for all $\xi$. Denote the set of all such $\rho$ by $S_{1}$. For $\rho \in S_{1}$ we have

$$
\int_{\xi_{1}-A}^{\xi_{1}+A} f(T) \mathrm{d} \xi \geqslant C f_{0} \int_{\xi_{1}-A}^{\xi_{1}+A} \omega^{-2}(\rho, \xi) \mathrm{d} \xi
$$

and so

$$
\begin{aligned}
\int_{\xi_{1}-A}^{\xi_{1}+A} \frac{\partial T}{\partial \xi} \omega^{-1}(\rho, \xi) \mathrm{d} \xi \mid & \leqslant C f_{0}^{-1 / 2}\left(\int_{\xi_{1}-A}^{\xi_{1}+A} f(T) \mathrm{d} \xi\right)^{1 / 2}\left(\int_{\xi_{1}-A}^{\xi_{1}+A} T_{\xi}^{2} \mathrm{~d} \xi\right)^{1 / 2} \\
& \leqslant C f_{0}^{-1 / 2} v_{0}^{-1} \int_{\xi_{1}-A}^{\xi_{1}+A}\left[\kappa|\nabla T|^{2}+\frac{v_{0}^{2}}{\kappa} f(T)\right] E_{1} E_{2} \mathrm{~d} \xi .
\end{aligned}
$$


The second case is that there exists $\xi_{4} \in\left[\xi_{1}-A, \xi_{1}+A\right]$ such that $T\left(\rho, \xi_{4}\right) \notin\left[\theta_{1}-\right.$ $\left.\zeta, \theta_{4}+\zeta\right]$. Denote the set of all such $\rho$ by $S_{2}$. For $\rho \in S_{2}$, we can find $\xi_{5}$ and $\xi_{6}$ such that $\left|T\left(\rho, \xi_{5}\right)-T\left(\rho, \xi_{6}\right)\right|=\zeta / 2$, and $T(\rho, \zeta) \in\left[\theta_{1}-\zeta, \theta_{4}+\zeta\right]$ for all $\xi$ between $\xi_{5}$ and $\xi_{6}$. In this case, similarly to the above reasoning, we have

$$
\int_{\xi_{1}-A}^{\xi_{1}+A} f(T(\rho, \xi)) \mathrm{d} \xi \int_{\xi_{1}-A}^{\xi_{1}+A} T_{\xi}^{2}(\rho, \xi) \mathrm{d} \xi \geqslant C \zeta^{2} f_{0}
$$

and so

$$
f_{0}^{-1 / 2} \zeta^{-1} v_{0}^{-1} \int_{\xi_{1}-A}^{\xi_{1}+A}\left[\kappa|\nabla T|^{2}+\frac{v_{0}^{2}}{\kappa} f(T)\right] E_{1} E_{2} \mathrm{~d} \xi \geqslant C .
$$

Then (6.8) implies that for $\rho \in S_{2}$ we have

$$
\left|\int_{\xi_{1}-A}^{\xi_{1}+A} \mathrm{~d} \xi_{3} \frac{\partial T}{\partial \xi} \omega^{-1}\left(\xi_{3}, \rho\right)\right| \leqslant C\left(1+\frac{A}{h}\right) f_{0}^{-1 / 2} \zeta^{-1} v_{0}^{-1} \int_{\xi_{1}-A}^{\xi_{1}+A}\left[\kappa|\nabla T|^{2}+\frac{v_{0}^{2}}{\kappa} f(T)\right] E_{1} E_{2} \mathrm{~d} \xi .
$$

Combining the two estimates (6.10) and (6.11), and taking into account that $A \geqslant h$, we obtain the result of Lemma 8.

Now we can finish the proof of Theorem 9. Taking into account (6.6) and (6.7) we see that

$$
\begin{aligned}
& \int_{\xi_{0}-A}^{\xi_{1}+A} \mathrm{~d} \xi \int_{h}^{3 h} \mathrm{~d} \rho G(h, \rho-2 h) E_{1} E_{2} T_{t}(\rho, \xi) \\
& \quad+C f_{0}^{-1 / 2} \zeta^{-1} \frac{\kappa}{v_{0} h} \int_{\xi_{0}-A}^{\xi_{1}+A} \mathrm{~d} \xi \int_{h}^{3 h} \mathrm{~d} \rho E_{1} E_{2}\left[\kappa|\nabla T|^{2}+\frac{v_{0}^{2}}{\kappa} f(T)\right] \\
& \geqslant \frac{1}{4 A^{2}} \int_{\xi_{0}-A}^{\xi_{0}+A} \mathrm{~d} \xi_{2} \int_{\xi_{1}-A}^{\xi_{1}+A} \mathrm{~d} \xi_{3} \int_{h}^{3 h} \mathrm{~d} \rho k(\rho)\left(T\left(\rho, \xi_{2}\right)-T\left(\rho, \xi_{3}\right)\right),
\end{aligned}
$$

and this implies

$$
\int_{\xi_{0}-A}^{\xi_{1}+A} \int_{h}^{3 h}\left(T_{t}+\kappa|\nabla T|^{2}+\frac{v_{0}^{2}}{\kappa} f(T)\right) E_{1} E_{2} \mathrm{~d} \xi \mathrm{d} \rho \geqslant C\left(f_{0}, \zeta\right)\left(1+\frac{l}{h}\right)^{-1}\left|\langle T\rangle_{\xi_{0}}-\langle T\rangle_{\xi_{1}}\right| F .
$$

\section{Cellular flows: Diffusive estimate}

Our goal in this section is to estimate the burning rate from below in terms of the jump of the temperature across the interface separating two cells. We will only consider this 
estimate in the context of the particular cellular flow we are studying, though it can be easily extended to a more general situation. Consider two neighboring cells, which we denote $C_{n-1}$ and $C_{n}$. We will look at two regions $D_{2 n-1} \subset C_{n-1}$ and $D_{2 n} \subset C_{n}$ which are symmetric under reflection with respect to the line separating the cells (see Fig. 5). The region $D_{2 n}$ is bounded by the curves $\rho=h, \rho=3 h, \xi=-A$, and $\xi=A$. For simplicity, we choose $\rho$ and $\xi$ coordinates in cell $C_{n-1}$ so that $\rho$ changes from $-3 h$ to $-h$ and $\xi$ from $-A$ to $A$ on $D_{2 n-1}$ (see the figure). Notice that this is different from the choice of coordinates we employed locally in each cell in the previous section; in terms of the old coordinates, $D_{2 n-1} \subset C_{n-1}$ is bounded by the curves $\rho=h, \rho=3 h, \xi=L-A$, $\xi=L+A$. With the new choice of coordinates, we denote by $I_{n}$ the part of $C_{n-1} \cup C_{n}$ bounded by the curves $\rho=-3 h, \rho=3 h, \xi=-A$, and $\xi=A$. We also denote, in the extension of notation of the previous section,

$$
\begin{aligned}
\langle T\rangle_{2 n-1} & =\frac{1}{2 A F} \int_{D_{2 n-1}} T(\rho, \xi) k(\rho) \mathrm{d} \xi \mathrm{d} \rho, \\
\langle T\rangle_{2 n} & =\frac{1}{2 A F} \int_{D_{2 n}} T(\rho, \xi) k(\rho) \mathrm{d} \xi \mathrm{d} \rho
\end{aligned}
$$

$(k(\rho)$ is defined in a natural way as $k(-\rho)$ when $\rho<0)$.

THEOREM 10. - There exist constants $C_{1,2}$ depending only on the constants in the bounds of Proposition 2, such that

$$
\kappa \int_{I_{n}}|\nabla T|^{2} \mathrm{~d} x \mathrm{~d} y \geqslant \frac{C_{1} \kappa H}{h}\left(\langle T\rangle_{2 n}-\langle T\rangle_{2 n-1}\right)^{2}
$$

for $A=C_{2} H$.

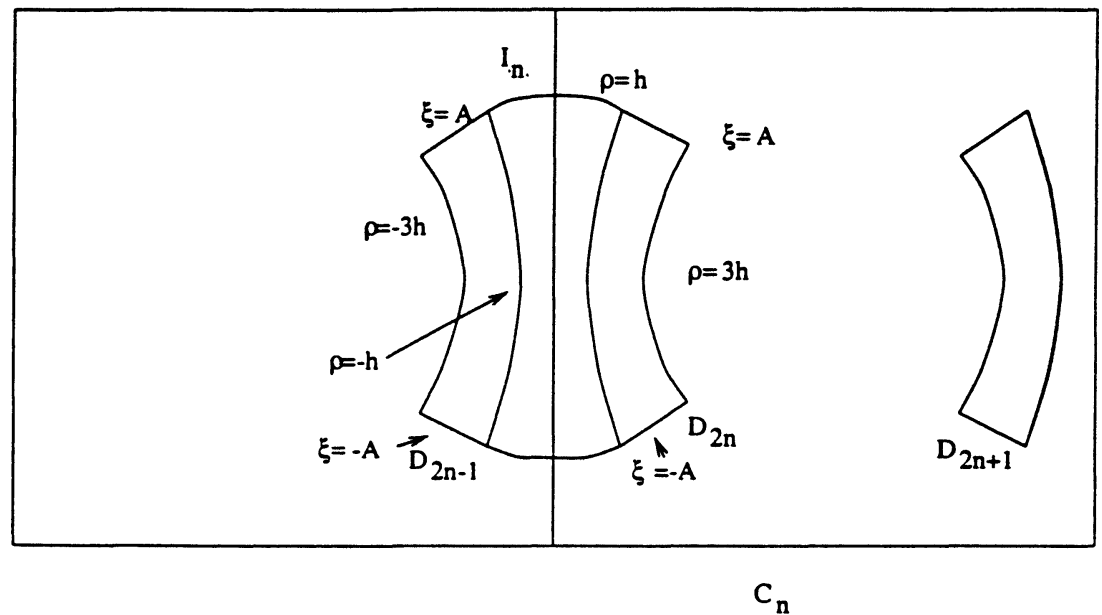

Fig. 5. The regions $D_{n}$. 
Proof. - Notice that

$$
\begin{aligned}
& \frac{1}{2 A F}\left|\int_{-A}^{A} \mathrm{~d} \xi\left(\int_{h}^{3 h} k(\rho) T(\rho, \xi) \mathrm{d} \rho-\int_{-h}^{-3 h} k(\rho) T(\rho, \xi) \mathrm{d} \rho\right)\right| \\
& \leqslant \frac{1}{2 A F} \int_{-A}^{A} \mathrm{~d} \xi \int_{h}^{3 h} k(\rho) \mathrm{d} \rho \int_{-3 h}^{3 h}\left|\frac{\partial T}{\partial \rho}(\gamma, \xi)\right| \mathrm{d} \gamma \\
& \leqslant\left(\frac{3 h}{A}\right)^{1 / 2}\left(\int_{-A}^{A} \int_{-3 h}^{3 h}\left|\frac{\partial T}{\partial \rho}(\rho, \xi)\right|^{2} \mathrm{~d} \rho\right)^{1 / 2} \leqslant C \frac{h^{1 / 2}}{H^{1 / 2}}\left(\int_{I_{n}}|\nabla T|^{2} \mathrm{~d} x \mathrm{~d} y\right) .
\end{aligned}
$$

Comparing the quantity we estimated with the definitions (7.1) and (7.2), we see that (7.3) follows.

\section{Cellular flows: Reactive estimate}

It is worthy to note that the diffusive estimate we proved in the last section is quadratic in the drop of the temperature over the interface. With the estimates we currently have we could not prove any lower bound for the burning rate, since the temperature could stay constant inside the cells (so that advective estimate does not give us anything) and drop in extremely small increments over the diffusive interfaces. There is no lower bound for such scenario because of the quadratic dependence. But in this case, the region where $T \in\left[\theta_{1}, \theta_{4}\right]$ would be very large, and hence we can hope to get a lower bound on $\int f(T)$. The following theorem is a rigorous expression of the above idea. We are mostly interested in the case $H \geqslant \kappa / v_{0}$ but include the case $H \leqslant \kappa / v_{0}$ for completeness.

THEOREM 11. - Assume that in a given cell $C_{n}$,

$$
\frac{1}{F} \int_{h}^{3 h} T(\rho, \xi) \mathrm{d} \rho \in\left[\theta_{1}, \theta_{4}\right]
$$

for every $\xi$. Then

$$
\int_{C_{n}}\left[\kappa|\nabla T|^{2}+\frac{v_{0}^{2}}{\kappa} f(T)\right] \mathrm{d} x \mathrm{~d} y \geqslant C\left(f_{0}, \zeta\right) v_{0} H \min \left(1, \frac{H v_{0}}{\kappa}\right) .
$$

Proof. - The proof uses an argument we already used several times when proving advective estimate. Consider a region in $C_{n}$ bounded by the curves $\rho=h$ and $\rho=H / 2$. By mean value theorem, for every $\xi$, there exists $\rho_{0} \in[h, 3 h]$ where $T\left(\rho_{0}, \xi\right) \in\left[\theta_{1}, \theta_{4}\right]$. All $\xi$ fall into two cases. In the first case, $T(\rho, \xi) \in\left[\theta_{1}-\zeta, \theta_{4}+\zeta\right]$ for every $\rho$ in the region $\rho \in[h, H / 2]$. Then

$$
\frac{v_{0}^{2}}{\kappa} \int_{\kappa}^{H / 2} f(T(\rho, \xi)) \mathrm{d} \rho \geqslant \frac{f_{0} H v_{0}^{2}}{\kappa}
$$


In the second case, there also exists $\rho_{1} \in[h, H / 2]$ such that $T\left(\rho_{1}, \xi\right) \notin\left[\theta_{1}-\zeta, \theta_{4}+\zeta\right]$. In this case we can find $\rho_{2}, \rho_{3}$ such that $\left|T\left(\rho_{2}, \xi\right)-T\left(\rho_{3}, \xi\right)\right| \geqslant \zeta$, and for every $\rho$ between $\rho_{2}$ and $\rho_{3}$, we have $T(\rho, \xi) \in\left[\theta_{1}-\zeta, \theta_{4}+\zeta\right]$. In this case, by the usual argument, we have

$$
f_{0}^{1 / 2} \zeta \leqslant\left(\int_{h}^{H / 2} f(T) \mathrm{d} \rho \int_{h}^{H / 2}|\nabla T|^{2} \mathrm{~d} \rho\right)^{1 / 2} \leqslant \frac{1}{2 v_{0}} \int_{h}^{H / 2}\left[\kappa|\nabla T|^{2}+\frac{v_{0}^{2}}{\kappa} f(T)\right] \mathrm{d} \rho .
$$

It is easy to show following Lemma 7 and Proposition 2 that the length of the interval of integration in $\xi, 2 L$, satisfies $2 L \geqslant C H$, where $C$ is some universal constant. Integrating over $\xi$, we then get the result of the theorem.

\section{Cellular flows: Putting it together}

Now we have all necessary estimates to establish the lower bound on burning rate in the case of cellular flows. We begin with an auxiliary computation. Its goal is to choose the right value of $h$ depending on other physical parameters fixed in the problem. The argument below may not be completely rigorous; we will fill in the gaps in the actual proof. Consider the sequence of averages (7.1), (7.2) introduced in Section 7 devoted to the diffusive estimate:

$$
\ldots,\langle T\rangle_{2 n},\langle T\rangle_{2 n+1},\langle T\rangle_{2(n+1)},\langle T\rangle_{2 n+3}, \ldots
$$

where $n$ varies from $-\infty$ to $\infty$. The values of the averages tend to 1 as $n \rightarrow-\infty$ and to 0 as $n \rightarrow \infty$. Assume that (for sufficiently large $U$ ) the change is gradual, and there exists a number of consecutive numbers $n$ where all averages lie in the interval $\left[\theta_{1}, \theta_{4}\right]$. Moreover, assume that in all cells corresponding to these values of $n$, we have

$$
\frac{1}{F} \int_{h}^{3 h} k(\rho) T(\rho, \xi) \mathrm{d} \rho \in\left[\theta_{1}, \theta_{4}\right]
$$

for all $\xi$, so that the reactive and linear advective estimates may be applied in these cells. It does not concern us here that $h$ has to be chosen yet; as we mentioned above, this is an auxiliary computation and the rigorous argument will appear in the proof. Let us denote

$$
\delta T_{n, \alpha}=\left|\langle T\rangle_{2 n+1}-\langle T\rangle_{2 n}\right|
$$

the change of the averages controlled by the advective estimate, and

$$
\delta T_{n, d}=\left|\langle T\rangle_{2(n+1)}-\langle T\rangle_{2 n+1}\right|
$$

the drop of the temperature controlled by diffusive estimate. We also set $\delta T_{n}=\delta T_{n, a}+$ $\delta T_{n, d}$ which we call the total drop over $n$th cell. Then we have, according to the advective 
estimate (6.3),

$$
\int_{C_{n}}\left(T_{t}+\kappa|\nabla T|^{2}+\frac{v_{0}^{2}}{\kappa} f(T)\right) \frac{\mathrm{d} x \mathrm{~d} y}{H} \geqslant C\left(f_{0}, \zeta\right)\left(1+\frac{\kappa}{v_{0} h}\right)^{-1} \frac{U h}{H} \delta T_{n, a},
$$

where the constant $C$ depends only on $f_{0}, \zeta$ and fixed constants associated with the geometry of streamlines of the flow. According to the diffusive estimate (7.3), we also have

$$
\int_{C_{n} \cup C_{n+1}}\left(\kappa|\nabla T|^{2}+\frac{v_{0}^{2}}{\kappa} f(T)\right) \frac{\mathrm{d} x \mathrm{~d} y}{H} \geqslant C \frac{\kappa}{h} \delta T_{n, d}^{2} .
$$

Also for every $C_{n}$ from the region we consider, we have by the reactive estimate (8.1) that

$$
\int_{C_{n}}\left(\kappa|\nabla T|^{2}+\frac{v_{0}^{2}}{\kappa} f(T)\right) \frac{\mathrm{d} x \mathrm{~d} y}{H} \geqslant C\left(f_{0}, \zeta\right) v_{0} \min \left(1, \frac{H v_{0}}{\kappa}\right)=C\left(f_{0}, \zeta\right) v_{0}
$$

under the assumption $H v_{0} / \kappa>1$ (see (4.5)). Fix some cell $C_{n}$, and introduce a parameter $0 \leqslant a_{n} \leqslant 1$ such that

$$
a_{n} \delta T_{n}=\delta T_{n, d}, \quad\left(1-a_{n}\right) \delta T_{n}=\delta T_{n, a} .
$$

According to (9.1), (9.2), and (9.3), we have

$$
\begin{aligned}
& \int_{C_{n} \cup C_{n+1}}\left(\kappa|\nabla T|^{2}+\frac{v_{0}^{2}}{\kappa} f(T)\right) \frac{\mathrm{d} x \mathrm{~d} y}{H} \\
& \geqslant C\left[\left(1+\frac{\kappa}{v_{0} h}\right)^{-1} \frac{U h}{H} \delta T_{n}\left(1-a_{n}\right)+\frac{\kappa a_{n}^{2}}{h} \delta T_{n}^{2}+v_{0}\right] .
\end{aligned}
$$

Over our sequence of cells, the value of temperature averages falls by a fixed amount, $\theta_{4}-\theta_{1}$. We are going to assume that this fall is gradual, and consider the lower bound on the contribution of the cells $C_{n}$ and $C_{n+1}$ to the total burning rate normalized by the temperature falloff $\delta T_{n}$ over the cell $C_{n}$. Namely, denote

$$
V_{n}=\int_{C_{n} \cup C_{n+1}}\left(T_{t}+\kappa|\nabla T|^{2}+\frac{v_{0}^{2}}{\kappa} f(T)\right) \frac{\mathrm{d} x \mathrm{~d} y}{H},
$$

then the burning rate $V$ satisfies

$$
V \geqslant \frac{1}{6} \sum_{n} V_{n}
$$

Eq. (9.4) may be rewritten as

$$
V_{n} \geqslant C\left[\left(1+\frac{\kappa}{v_{0} h}\right)^{-1} \frac{U h}{H}\left(1-a_{n}\right)+\frac{\kappa a_{n}^{2}}{h} \delta T_{n}+\frac{v_{0}}{\delta T_{n}}\right] \delta T_{n} .
$$


Moreover,

$$
\sum \delta T_{n} \geqslant \theta_{4}-\theta_{1}
$$

where summation is taken over the set of cells where all estimates apply. In the lower bound (9.5), $a_{n}$ and $\left|\delta T_{n}\right|$ are out of our control, but we may choose $h$ optimally. Hence if we denote

$$
\begin{aligned}
& B\left(\kappa, v_{0}, U, H\right) \\
& \quad=\max _{0 \leqslant h \leqslant H / 6}\left\{\min _{0 \leqslant a_{n} \leqslant 1,0 \leqslant \delta T_{n} \leqslant 2}\left(\left(1+\frac{\kappa}{v_{0} h}\right)^{-1} \frac{U h}{H}\left(1-a_{n}\right)+\frac{\kappa a_{n}^{2}}{h} \delta T_{n}+\frac{v_{0}}{\delta T_{n}}\right)\right\},
\end{aligned}
$$

then

$$
V \geqslant C B\left(\kappa, v_{0}, U, H\right)\left(\theta_{4}-\theta_{1}\right) .
$$

Therefore our goal is to find $B\left(\kappa, v_{0}, U, H\right)$. The minimum in $\delta T_{n}$ is achieved when

$$
\delta T_{n}=\min \left(2, \frac{1}{a_{n}} \sqrt{\frac{v_{0} h}{\kappa}}\right) .
$$

Let us consider two different regimes.

(1) If $\kappa / v_{0} h \leqslant 1$ then the minimum is achieved for $\delta T_{n} \geqslant 1$ and

$$
B\left(\kappa, v_{0}, U, H\right) \geqslant C \max _{0 \leqslant h \leqslant H / 3}\left\{\min _{0 \leqslant a_{n} \leqslant 1}\left(\frac{U h}{H}\left(1-a_{n}\right)+\frac{\kappa a_{n}^{2}}{h}+v_{0}\right)\right\},
$$

where $C$ is a universal constant. Choose $h$ out of the condition $U h / H=\kappa / h$, so that

$$
h=\sqrt{\frac{\kappa H}{U}} .
$$

Then evidently

$$
B\left(\kappa, v_{0}, U, H\right) \geqslant C\left(\sqrt{\frac{\kappa U}{H}}+v_{0}\right)=C v_{0}\left(\sqrt{\frac{\tau_{c}}{\tau_{u}}}+1\right)
$$

with $\tau_{c}$ and $\tau_{u}$ defined in (4.6). Given (9.6), condition $\kappa / v_{0} h \leqslant 1$ translates into

$$
\frac{1}{v_{0}} \sqrt{\frac{\kappa U}{H}}=\frac{\tau_{c}}{\tau_{u}} \leqslant 1
$$

For this computation to apply, we also need to ensure that $h \leqslant H / 6$, that is,

$$
\sqrt{\frac{\kappa}{U H}} \leqslant 1 / 6,
$$

so that $h$ is in the acceptable range. This is a condition on the Peclet number. All our bounds will remain valid if replace the choice of $h$ by $C h$ with $C$ in some fixed range; 
we just have to adjust the universal constants. Therefore, for presentation purposes we will henceforth require the condition (4.4)

$$
\mathrm{Pe}=\sqrt{\frac{U H}{\kappa}} \geqslant 1 .
$$

(2) If $\frac{\kappa}{v_{0} h} \geqslant 1$ then, since $\delta T_{n}=\min \left(2, \frac{1}{a_{n}} \sqrt{\frac{v_{0} h}{\kappa}}\right)$, we have that

$$
B\left(\kappa, v_{0}, U, H\right) \geqslant \max _{0 \leqslant h \leqslant H / 6} \min _{0 \leqslant a_{n} \leqslant 1} g\left(a_{n}\right),
$$

where

$$
g\left(a_{n}\right)= \begin{cases}\frac{U h^{2} v_{0}}{\kappa H}\left(1-a_{n}\right)+\frac{2 \kappa a_{n}^{2}}{h}+\frac{v_{0}}{2}, & a_{n} \leqslant \frac{1}{2} \sqrt{\frac{v_{0} h}{\kappa}}, \\ \frac{U h^{2} v_{0}}{\kappa H}\left(1-a_{n}\right)+2 a_{n} \sqrt{\frac{v_{0} \kappa}{h}}, & a_{n} \geqslant \frac{1}{2} \sqrt{\frac{v_{0} h}{\kappa}} .\end{cases}
$$

Note that

$$
\begin{aligned}
& \max _{0 \leqslant h \leqslant H / 6} \min _{0 \leqslant a_{n} \leqslant 1}\left(\frac{U h^{2} v_{0}}{\kappa H}\left(1-a_{n}\right)+\frac{2 \kappa a_{n}^{2}}{h}+\frac{v_{0}}{2}\right) \\
& \geqslant C\left(\left(U v_{0} H^{-1} \kappa\right)^{1 / 3}+v_{0}\right)=C v_{0}\left(\left(\frac{\tau_{c}}{\tau_{u}}\right)^{1 / 3}+1\right),
\end{aligned}
$$

since we can choose $h$ from $U h^{2} v_{0} /(\kappa H)=\kappa / h$, which gives

$$
h=\left(\frac{\kappa^{2} H}{u v_{0}}\right)^{1 / 3} \text {. }
$$

Also

$$
\max _{0 \leqslant h \leqslant H / 3} \min _{0 \leqslant a_{n} \leqslant 1}\left(\frac{U h^{2} v_{0}}{\kappa H}\left(1-a_{n}\right)+2 a_{n} \sqrt{\frac{v_{0} \kappa}{h}}\right) \geqslant C\left(U v_{0}^{3} \kappa H^{-1}\right)^{1 / 5}=C v_{0}\left(\frac{\tau_{c}}{\tau_{u}}\right)^{1 / 5},
$$

since we can choose $h$ from $U h^{2} v_{0} /(\kappa H)=\sqrt{v_{0} \kappa / h}$, which gives

$$
h=\left(\frac{\kappa^{3} H^{2}}{U^{2} v_{0}}\right)^{1 / 5} \text {. }
$$

Therefore,

$$
\max _{0 \leqslant h \leqslant H / 3} \min _{0 \leqslant a_{n} \leqslant 1} g\left(a_{n}\right) \geqslant C v_{0} \min \left\{\left(\left(\frac{\tau_{c}}{\tau_{u}}\right)^{1 / 3}+1\right),\left(\frac{\tau_{c}}{\tau_{u}}\right)^{1 / 5}\right\} .
$$

Notice that $\tau_{c} / \tau_{u} \geqslant 1$ in the regime we consider, since $\tau_{c} / \tau_{u}$ is also equal to $\left(\kappa / v_{0} h\right)^{3}$ with $h$ chosen according to $(9.7)$ or $\left(\kappa / v_{0} h\right)^{5 / 2}$ with $h$ chosen according to (9.8). 
Therefore, we get that the second regime is characterized by

$$
\frac{\tau_{c}}{\tau_{u}}=\frac{1}{v_{0}} \sqrt{\frac{\kappa U}{H}} \geqslant 1,
$$

and in this case

$$
B\left(\kappa, v_{0}, U, H\right) \geqslant C v_{0}\left(\frac{\tau_{c}}{\tau_{u}}\right)^{1 / 5} .
$$

The choice of $h$ is given by (9.8). In this regime, we also need to assume that

$$
\left(\frac{\kappa^{3}}{U^{2} H^{3} v_{0}}\right)^{1 / 5} \leqslant 1 / 6
$$

so that $h$ is in the acceptable range. This, up to a constant, is a condition

$$
\frac{l}{H} \leqslant \mathrm{Pe}^{2}
$$

which is satisfied provided (4.5) and (4.4) hold.

Now we are finally ready to give a proof of Theorem 7 .

Proof. - Given the parameters $\kappa, v_{0}, H$ and $U$, define $h$ according to

$$
\begin{aligned}
& h=\frac{1}{6} \sqrt{\frac{\kappa H}{U}}, \quad \tau_{c} / \tau_{u} \leqslant 1, \\
& h=\frac{1}{6}\left(\frac{\kappa^{3} H^{2}}{U^{2} v_{0}}\right)^{1 / 5}, \quad \tau_{c} / \tau_{u} \geqslant 1 .
\end{aligned}
$$

These choices ensure that $h \leqslant H / 6$ provided that (4.4), (4.5) are satisfied. Consider the smallest integer number $m_{1}$ such that $\langle T\rangle_{i} \leqslant \theta_{4}-\left(\theta_{4}-\theta_{1}\right) / 10$ for all $i \geqslant m_{1}$. We need to consider several options. The first is taken care of by

Lemma 9. - If $\langle T\rangle_{m_{1}} \leqslant \theta_{4}-\left(\theta_{4}-\theta_{1}\right) / 5$ then the bounds of Theorem 7 hold. Moreover, the same is true if for some $m, m+1$, we have $\langle T\rangle_{m},\langle T\rangle_{m+1} \in\left[\theta_{1}, \theta_{4}\right]$ and

$$
\left|\langle T\rangle_{m+1}-\langle T\rangle_{m}\right| \geqslant\left(\theta_{4}-\theta_{1}\right) / 10 .
$$

Proof. - Let us consider the case where $\langle T\rangle_{m_{1}} \leqslant \theta_{4}-\left(\theta_{4}-\theta_{1}\right) / 5$, the other case is similar. If $m_{1}=2 n$ is even, notice that by definition of $m_{1},\langle T\rangle_{2 n-1} \geqslant \theta_{4}-\left(\theta_{4}-\theta_{1}\right) / 10$. Then by the diffusive estimate (7.3) we obtain

$$
V \geqslant \frac{1}{3} V_{n} \geqslant C \frac{\kappa}{h}\left(\theta_{4}-\theta_{1}\right)^{2} .
$$

In the case where $\tau_{c} / \tau_{u} \leqslant 1$ putting in $h$ given by (9.9) gives exactly the bound (4.7) of Theorem 7, modulo $C_{2} v_{0}$. But by Proposition 1, we can always add $C v_{0}$ to the lower bound on $V$. In the case where $\tau_{c} / \tau_{u} \geqslant 1$, putting in $h$ given by (9.10) gives the lower 
bound $C\left(\kappa^{2} U^{2} H^{-2} v_{0}\right)^{1 / 5}=C v_{0}\left(\tau_{c} / \tau_{u}\right)^{2 / 5}$, which is even better than the bound (4.7) (modulo $v_{0}$ ). Hence, Theorem 7 also holds.

If $m_{1}=2 n+1$ is odd, then by definition of $m_{1},\langle T\rangle_{2 n} \geqslant \theta_{4}-\left(\theta_{4}-\theta_{1}\right) / 10$. Thus temperature falls inside the cell $C_{n}$, and we may use advective estimate of Theorem 8 . We can find $\xi_{0}, \xi_{1}$ in the cell $C_{n}$ such that

$$
\frac{1}{F} \int_{h}^{3 h} k(\rho) T\left(\rho, \xi_{0}\right) \mathrm{d} \rho=\theta_{4}-\frac{\theta_{4}-\theta_{1}}{10}, \quad \frac{1}{F} \int_{h}^{3 h} k(\rho) T\left(\rho, \xi_{1}\right) \mathrm{d} \rho=\theta_{4}-\frac{\theta_{4}-\theta_{1}}{5}
$$

and

$$
\frac{1}{F} \int_{h}^{3 h} k(\rho) T(\rho, \xi) \mathrm{d} \rho \in\left[\theta_{4}-\frac{\theta_{4}-\theta_{1}}{5}, \theta_{4}-\frac{\theta_{4}-\theta_{1}}{10}\right]
$$

for every $\xi$ between $\xi_{0}$ and $\xi_{1}$. By the advective estimate (6.2), we get

$$
V \geqslant \frac{1}{3} V_{n} \geqslant C\left(\zeta, f_{0}\right)\left(1+\frac{\kappa}{v_{0} h}\right)^{-1}\left(\theta_{4}-\theta_{1}\right)^{3} \frac{U h}{H}
$$

Direct substitution of the expression (9.9) or (9.10) for $h$ depending on the value of $\tau_{c} / \tau_{u}$ and comparison of the above bound with (4.7) gives the conclusion of Theorem 7 . The proof of the second statement of this lemma is parallel to the above argument.

Lemma 9 proves Theorem 7 unless there exists a sequence $m_{1}, \ldots, m_{2}$ (of length at least 8 , in fact) such that for every $m_{1} \leqslant m \leqslant m_{2}$.

$$
\langle T\rangle_{m} \in\left[\theta_{1}+\frac{\theta_{4}-\theta_{1}}{10}, \theta_{4}-\frac{\theta_{4}-\theta_{1}}{10}\right]
$$

and for $m=m_{1}, \ldots, m_{2}-1$

$$
\left|\langle T\rangle_{m+1}-\langle T\rangle_{m}\right| \leqslant \frac{\theta_{4}-\theta_{1}}{10} .
$$

Therefore there exists a sequence of at least three (or more) consecutive cells $C_{n_{1}}, \ldots, C_{n_{2}}$ such that and

$$
\langle T\rangle_{2 n_{1}} \geqslant \theta_{4}-\frac{3}{10}\left(\theta_{4}-\theta_{1}\right),\langle T\rangle_{2 n_{2}+1} \leqslant \theta_{1}+\frac{3}{10}\left(\theta_{4}-\theta_{1}\right),
$$

and

$$
\langle T\rangle_{m} \in\left[\theta_{4}-\frac{\theta_{4}-\theta_{1}}{10}, \theta_{1}+\frac{\theta_{4}-\theta_{1}}{10}\right]
$$


for every $m$ such that $2 n_{1} \leqslant m \leqslant 2 n_{2}+1$. Assume that there exists a cell $C_{n}$ with $n_{1} \leqslant n \leqslant n_{2}$ where we can find $\xi_{0}$ so that

$$
\int_{h}^{3 h} k(\rho) T\left(\rho, \xi_{0}\right) \mathrm{d} \rho \notin\left[\theta_{1}, \theta_{4}\right] .
$$

By (9.12) and mean value theorem we can also find $\xi_{1}$ in this cell such that

$$
\int_{h}^{3 h} k(\rho) T\left(\rho, \xi_{1}\right) \mathrm{d} \rho \in\left[\theta_{1}+\frac{\theta_{4}-\theta_{1}}{10}, \theta_{4}-\frac{\theta_{4}-\theta_{1}}{10}\right] .
$$

Therefore, the advective estimate (6.2) can be applied in this cell, giving

$$
V_{n} \geqslant C\left(\zeta, f_{0}\right)\left(1+\frac{\kappa}{v_{0} h}\right)^{-1}\left(\theta_{4}-\theta_{1}\right)^{3} \frac{U h}{H}
$$

Hence, similarly to the proof of Lemma 9 . Theorem 7 holds in this case. The only case left to consider is the case where for every $n_{1} \leqslant n \leqslant n_{2}$, for every $\xi$ in a cell $C_{n}$, we have

$$
\int_{h}^{3 h} k(\rho) T(\rho, \xi) \mathrm{d} \rho \in\left[\theta_{1}, \theta_{4}\right] .
$$

In this case the second advective estimate (6.3), as well as reactive estimate (8.1) apply in every cell $C_{n}$ such that $n_{1} \leqslant n_{2}$. Recall the notation

$$
\begin{aligned}
& \left(1-a_{n}\right) \delta T_{n}=\delta T_{n, a}=\left|\langle T\rangle_{2 n+1}-\langle T\rangle_{2 n}\right|, \\
& a_{n} \delta T_{n}=\delta T_{n, d}=\left|\langle T\rangle_{2(n+1)}-\langle T\rangle_{2 n+1}\right| .
\end{aligned}
$$

Following the computation we performed at the beginning of this section we get

$$
V \geqslant \frac{1}{6} \sum_{n=n_{1}}^{n_{2}} V_{n} \geqslant C \sum_{n=n_{1}}^{n_{2}}\left[\left(\left(1+\frac{\kappa}{v_{0} h}\right)^{-1} \frac{U h}{H}\left(1-a_{n}\right)+\frac{\kappa}{h} a_{n}^{2} \delta T_{n}+v_{0} \delta T_{n}^{-1}\right) \delta T_{n}\right] .
$$

Consider the case where $\tau_{c} / \tau_{u} \geqslant 1$ (the other case is similar but simpler). Putting the expression for $h$ from (9.10) into (9.13), we get

$$
V \geqslant C \sum_{n=n_{1}}^{n_{2}}\left(\left[v_{0}\left(\frac{\tau_{c}}{\tau_{u}}\right)^{1 / 5}\left(1-a_{n}\right)+v_{0}\left(\frac{\tau_{c}}{\tau_{u}}\right)^{2 / 5} a_{n}^{2} \delta T_{n}+v_{0} \delta T_{n}^{-1}\right] \delta T_{n}\right) .
$$

Since by (9.11), $\sum_{n=n_{1}}^{n_{2}} \delta T_{n} \geqslant 2\left(\theta_{4}-\theta_{1}\right) / 5$, we have

$$
V \geqslant C v_{0} \min _{0 \leqslant a_{n} \leqslant 1,0 \leqslant \delta T_{n} \leqslant 2}\left[\left(\frac{\tau_{c}}{\tau_{u}}\right)^{1 / 5}\left(1-a_{n}\right)+\left(\frac{\tau_{c}}{\tau_{u}}\right)^{2 / 5} a_{n}^{2} \delta T_{n}+\delta T_{n}^{-1}\right] .
$$


It remains to show that the expression in square brackets in (9.14) is always greater or equal to $C v_{0}\left(\tau_{c} / \tau_{u}\right)^{1 / 5}$ (we can always add $C v_{0}$ later to the lower bound for $V$ by Proposition 1). If $a_{n}<1 / 2$, the first term in the sum gives exactly the estimate we need (no matter what is the value of $\delta T_{n} \leqslant 1$ ). Hence it remains to consider the case where $a_{n}>1 / 2$. In this case the sum of the second and third term in the square brackets is greater than or equal to

$$
C v_{0}\left(\left(\frac{\tau_{c}}{\tau_{u}}\right)^{2 / 5} \delta T_{n}+\delta T_{n}^{-1}\right) \geqslant C v_{0}\left(\frac{\tau_{c}}{\tau_{u}}\right)^{1 / 5}
$$

and this finishes the proof of Theorem 7.

\section{Acknowledgement}

We wish to thank Peter Constantin for initiating our collaboration on the subject, and for numerous useful discussions and advice. We are grateful to Henri Berestycki, Fausto Cattaneo, Albert Fannjiang, Francois Hamel and George Papanicolaou for interesting discussions. AK was supported by NSF grant DMS-9801530, and LR was supported by NSF grant DMS-9971742 and ASCI Flash Center at the University of Chicago.

\section{REFERENCES}

[1] Audoly B., Berestycki H., Pomeau Y., Reaction-diffusion in fast steady flow, C.R. Acad. Sci., Ser. IIB, 328, 255-262.

[2] Bensoussan A., Lions J.L., Papanicolaou G., Asymptotic Analysis for Periodic Structures, North-Holland, Amsterdam, 1978.

[3] Berestycki H., Hamel F., Propagation of flames through vortical cells, preprint.

[4] Berestycki H., Larrouturou B., Lions P.L., Multi-dimensional traveling wave solutions of a flame propagation model, Arch. Rational Mech. Anal 111 (1990) 33-49.

[5] Berestycki H., Larrouturou B., Roquejoffre J.-M., Stability of traveling fronts in a model for flame propagation I: linear stability, Arch. Rational Mech. Anal. 117 (1992) 97-117.

[6] Berestycki H., Nirenberg L., Some qualitative properties of solutions of semilinear equations in cylindrical domains, in: Rabinowitz P. et al. (Eds.), Analysis etc., Academic Press, 1990, pp. 115-164.

[7] Berestycki H., Nirenberg L., Traveling fronts in cylinders, Annales de l'IHP, Analyse non linéare 9 (1992) 497-572.

[8] Clavin P., Williams F.A., Theory of pre-mixed flame propagation in large-scale turbulence, J. Fluid. Mech. 90 (1979) 589-604.

[9] Constantin P., Kiselev A., Oberman A., Ryzhik L., Bulk burning rate in passive-reactive diffusion, Arch. Rat. Mech. Anal. 154 (2000) 53-91.

[10] Embid P., Majda A., Souganidis P., Comparison of turbulent flame speeds from complete averaging and the G-equation, Phys. Fluids. 7 (1995) 2052-2060.

[11] Embid P., Majda A., Souganidis P., Effective geometric from dynamics for premixed turbulent combustion with separated velocity scales, Combust. Sci. Tech. 103 (1994) 85.

[12] Fannjiang A., Papanicolaou G., Convection enhanced diffusion for periodic flows, SIAM J. Appl. Math. 54 (1994) 333-408.

[13] Fisher R., The wave of advance of advantageous genes, Ann. Eugenics 7 (1937) 355-369. 
[14] Freidlin M., Gärtner J., On the propagation of concentration waves in periodic and random media, Soviet Math. Dokl. 20 (1979) 1282-1286.

[15] Freidlin M., Geometric optics approach to reaction-diffusion equations, SIAM J. Appl. Math. 46 (1986) 222-232.

[16] Freidlin M., Limit theorems for large deviations and reaction-diffusion equations, Ann. Probab. 13 (1985) 639-675.

[17] Hamel F., Formules min-max pour les vitesses d'ondes progressives multidimensionnelles, Ann. Fac. Sci., Toulouse Math. (6) 8 (1999) 259-280.

[18] Heinze S., Papanicolaou G., Stevens A., Variational principles for propagation speeds in inhomogeneous media, SIAM J. Appl. Math., to appear.

[19] Kerstein A., Ashurst W., Phys. Rev. Lett. 68 (1992) 934.

[20] Kerstein A., Simple derivation of Yakhot's turbulent premixed flame speed formula, Combust. Sci. Tech. 60 (1988) 163-165.

[21] Kagan L., Sivashinsky G., Makhviladze G., On flame extinction by a spatially periodic shear flow, Combust. Theor. Model. 2 (1998) 399-404.

[22] Kagan L., Sivashinsky G., On flame propagation and extinction in large scale vortical flows, Combustion and Flame 120 (2000) 222-232.

[23] Kolmogorov A.N., Petrovskii I.G., Piskunov N.S., Étude de I'équation de la chaleurde matiére et son application à un problème biologique, Bull. Moskov. Gos. Univ. Mat. Mekh. 1 (1937) 1-25 (see [27] pp. 105-130 for an English transl.).

[24] Majda A., Souganidis P., Large scale front dynamics for turbulent reaction-diffusion equations with separated velocity scales, Nonlinearity 7 (1994) 1-30.

[25] Mallordy J.-F., Roquejoffre J.-M., A parabolic equation of the KPP type in higher dimensions, SIAM J. Math. Anal. 26 (1995) 1-20.

[26] McLaughlin R., Zhu J., The effect of finite front thickness on the enhanced speed of propagation, Combust. Sci. Tech. 129 (1997) 89-112.

[27] Pelcé P. (Ed.), Dynamics of Curved Fronts, Academic Press, 1988.

[28] Protter M., Weinberger H., Maximum Principles in Differential Equations, Prentice-Hall, Englewood Cliffs, NJ, 1967.

[29] Ronney P., Some open issues in premixed turbulent combustion, in: Buckmuster J., Takeno T. (Eds.), Modeling in Combustion Science, Springer-Verlag, Berlin, 1995.

[30] Roquejoffre J.-M., Stability of traveling fronts in a model for flame propagation II: nonlinear stability, Arch. Rat. Mech. Anal. 117 (1992) 119-153.

[31] Xin J., Existence of planar flame fronts in convective-diffusive periodic media, Arch. Rat. Mech. Anal. 121 (1992) 205-233.

[32] Xin J., Existence and nonexistence of traveling waves and reaction-diffusion front propagation in periodic media, J. Stat. Phys. 73 (1993) 893-926.

[33] Xin J., Analysis and modeling of front propagation in heterogeneous media, SIAM Review 42 (2000) 161-230.

[34] Yakhot V., Propagation velocity of premixed turbulent flames, Combust. Sci. Tech. 60, 191214. 